\title{
Effects of Wall Cooling on Hypersonic Boundary Layer Receptivity over a Cone
}

\author{
K. Kara* \\ Department of Aerospace Engineering \\ Old Dominion University, Norfolk, VA, 23529 \\ P. Balakumar ${ }^{\dagger}$ \\ Flow Physics and Control Branch \\ NASA Langley Research Center, Hampton, VA 23581 \\ O. A. Kandil \\ Department of Aerospace Engineering \\ Old Dominion University, Norfolk, VA, 23529
}

\begin{abstract}
Effects of wall cooling on the receptivity process induced by the interaction of slow acoustic disturbances in the free-stream are numerically investigated for a boundary layer flow over a 5-degrees straight cone. The free-stream Mach number is 6.0 and the Reynolds number is $7.8 \times 10^{6} / \mathrm{ft}$. Both the steady and unsteady solutions are obtained by solving the full Navier-Stokes equations using $5^{\text {th }}$-order accurate weighted essentially non-oscillatory (WENO) scheme for space discretization and using $3^{\text {rd }}$-order total variation diminishing (TVD) Runge-Kutta scheme for time integration. Computations are performed for a cone with nose radius of 0.001 inch for adiabatic wall temperature $\left(T_{a w}\right), 0.75^{\star} T_{a w}, 0.5^{\star} T_{a w}$, $0.40^{\star} T_{\mathrm{aw}}, 0.30^{\star} \mathrm{T}_{\mathrm{aw}}$, and $0.20^{\star} \mathrm{T}_{\mathrm{aw}}$. 0 nce the mean flow field is computed, disturbances are introduced at the upstream end of the computational domain. Generation of instability waves from leading edge region and receptivity of boundary layer to slow acoustic waves are investigated. Computations showed that wall cooling has strong stabilization effect on the first mode disturbances as was observed in the experiments. Transition location moved to upstream when wall cooling was applied It is also found that the boundary layer is much more receptive to fast acoustic wave (by almost a factor of 50 ). When simulations performed using the same forcing frequency growth of the second mode disturbances are delayed with wall cooling and they attained values two times higher than that of adiabatic case. In $0.20^{*} T_{\text {aw }}$ case the transition Reynolds number is doubled compared to adiabatic conditions. The receptivity coefficient for adiabatic wall case $\left(804^{\circ} \mathrm{R}\right)$ is 1.5225 and for highly cooled cones $\left(241\right.$, and $\left.161^{\circ} \mathrm{R}\right)$; they are in the order of $10^{-3}$.
\end{abstract}

\section{Introduction}

$\mathrm{T}$ ransition process from laminar to turbulent flow is still an important challenge even after years of research. Accurate prediction of transition location is vital for the design of hypersonic vehicles. Because transition controls important quantities such as aerodynamic drag, heat transfer and other boundary layer parameters. In hypersonic boundary layers one important parameter is the wall temperature. Wall cooling would be expected to stabilize first mode disturbances while destabilizing the second mode. The effects of cooling on transition have been studied experimentally and numerically by many researchers.

Lees ${ }^{1}$ predicted that cooling the surface would stabilize the boundary layer. Later, Mack's ${ }^{2}$ results showed that the first mode was stabilized by cooling; however the higher modes were destabilized by this process. Experiments of Demetriades ${ }^{3}$ and Lysenko ${ }^{4}$ confirmed that cooling the wall increased the growth rates of the second mode

\footnotetext{
* Graduate Research Assistant, Student Member AIAA

${ }^{\dagger}$ Research Scientist, Member AIAA

${ }^{\star}$ Professor \& Eminent Scholar, Associate Fellow AIAA
} 
disturbances and reduced the transition Reynolds number. Stetson ${ }^{5}$ investigated the effects of surface temperature on the stability of the laminar boundary layer experimentally and the results also verified linear stability theory. Balakumar and Malik ${ }^{6}$ computed the parametric effects of the pressure gradient and wall cooling on the stability of the flow over cone using the quasi-parallel, compressible linear stability $\mathrm{e}^{\text {Malik }}$ code.

Receptivity $^{7}$ is a process by which free-stream or wall-induced disturbances enter the laminar boundary layer and generate unstable waves ${ }^{8}$. The receptivity process is poorly understood at hypersonic speeds. The understanding of this phenomenon is of great importance because receptivity connects the amplitude of the free-stream disturbances and initial amplitude of the unstable waves ${ }^{9}$. Recent experimental ${ }^{9-13}$, theoretical ${ }^{14-19}$ and computationa ${ }^{20-35}$ studies $^{2}$ increased our understanding about receptivity mechanism. However it still remains as a challenging problem with practical importance.

The objectives of this work are to estimate the destabilizing effect of the wall cooling on the hypersonic boundary layers over a blunt cone and to calculate the receptivity coefficient of the instability waves generated near the leading edge. To do so simulations are performed at different wall temperatures $\mathrm{T}_{\mathrm{aw}}$ (adiabatic wall temperature), $0.75 * \mathrm{~T}_{\mathrm{aw}}, 0.50 * \mathrm{~T}_{\mathrm{aw}}, 0.40 * \mathrm{~T}_{\mathrm{aw}}, 0.30 * \mathrm{~T}_{\mathrm{aw}}$ and $0.20 * \mathrm{~T}_{\mathrm{aw}}$ for leading edge nose radius $\mathrm{R}_{\mathrm{n}}=0.001$ inch and at a unit Reynolds number of $7.8 * 10^{6} / \mathrm{ft}$ for a 5 -degree half angle cone.

\section{Governing Equations}

The equations solved are the conservative unsteady compressible two-dimensional axisymmetric Navier-Stokes equations

$$
\frac{\partial Q}{\partial t}+\frac{\partial F}{\partial x}+\frac{\partial G}{\partial y}=\frac{\partial F_{v}}{\partial x}+\frac{\partial G_{v}}{\partial y}+S
$$

where $Q$ is the solution flow field vector, $F$ and $G$ are the axial- and radial-direction inviscid flux vectors given by

$$
Q=\left[\begin{array}{c}
\rho \\
\rho u \\
\rho V \\
\rho E
\end{array}\right] \quad F=\left[\begin{array}{c}
\rho u \\
\rho u^{2}+p \\
\rho u v \\
(\rho E+p) u
\end{array}\right] \quad G=\left[\begin{array}{c}
\rho v \\
\rho v u \\
\rho V^{2}+p \\
(\rho E+p) v
\end{array}\right]
$$

and $F_{v}$ and $G_{v}$ are the axial- and radial-direction viscous and heat conduction flux vectors given by

$$
F_{v}=\left[\begin{array}{c}
0 \\
\tau_{x x} \\
\tau_{x y} \\
u \tau_{x x}+v \tau_{x y}-q_{x}
\end{array}\right] \quad G_{v}=\left[\begin{array}{c}
0 \\
\tau_{y x} \\
\tau_{y y} \\
u \tau_{y x}+v \tau_{y y}-q_{y}
\end{array}\right]
$$

The vector $S$ contains viscous flux and source terms associated with the axisymmetric geometry. In twodimensional formulation, $\mathrm{S}$ will be equal to zero.

The source term, shear stresses and heat fluxes have the following form 


$$
\begin{aligned}
& S=\frac{1}{y}\left\{\left[\begin{array}{c}
0 \\
\tau_{y x} \\
\tau_{y y}-\tau_{\theta \theta} \\
u \tau_{y x}+v \tau_{y y}-q_{y}
\end{array}\right]-\left[\begin{array}{c}
\rho v \\
\rho u v \\
\rho v^{2} \\
(\rho E+p) v
\end{array}\right]\right\} \\
& \tau_{x x}=\frac{2}{3} \frac{\mu}{\operatorname{Re}}\left(2 \frac{\partial u}{\partial x}-\frac{\partial v}{\partial y}-\frac{v}{y}\right) \quad \tau_{y y}=\frac{2}{3} \frac{\mu}{\operatorname{Re}}\left(2 \frac{\partial v}{\partial y}-\frac{\partial u}{\partial x}-\frac{v}{y}\right) \\
& \tau_{\theta \theta}=\frac{2}{3} \frac{\mu}{\operatorname{Re}}\left(2 \frac{v}{y}-\frac{\partial u}{\partial x}-\frac{\partial v}{\partial y}\right) \quad \tau_{x y}=\frac{\mu}{\operatorname{Re}}\left(\frac{\partial u}{\partial y}+\frac{\partial v}{\partial x}\right) \\
& q_{x}=-\frac{\gamma}{(\gamma-1) \operatorname{Pr} \operatorname{Re}} \frac{\partial T}{\partial x} \quad q_{y}=-\frac{\gamma}{(\gamma-1) \operatorname{Pr} \operatorname{Re}} \frac{\partial T}{\partial y}
\end{aligned}
$$

There exist a singularity along the axis of symmetry $y=0$. After applying L'Hopital rule and taking limit as $y$ goes to zero using the symmetry conditions $\frac{\partial u}{\partial y}=\frac{\partial \rho}{\partial y}=\frac{\partial T}{\partial y}=\frac{\partial p}{\partial y}=0, v=0$ at $\mathrm{y}=0$, source term along the axis $y=0$ becomes

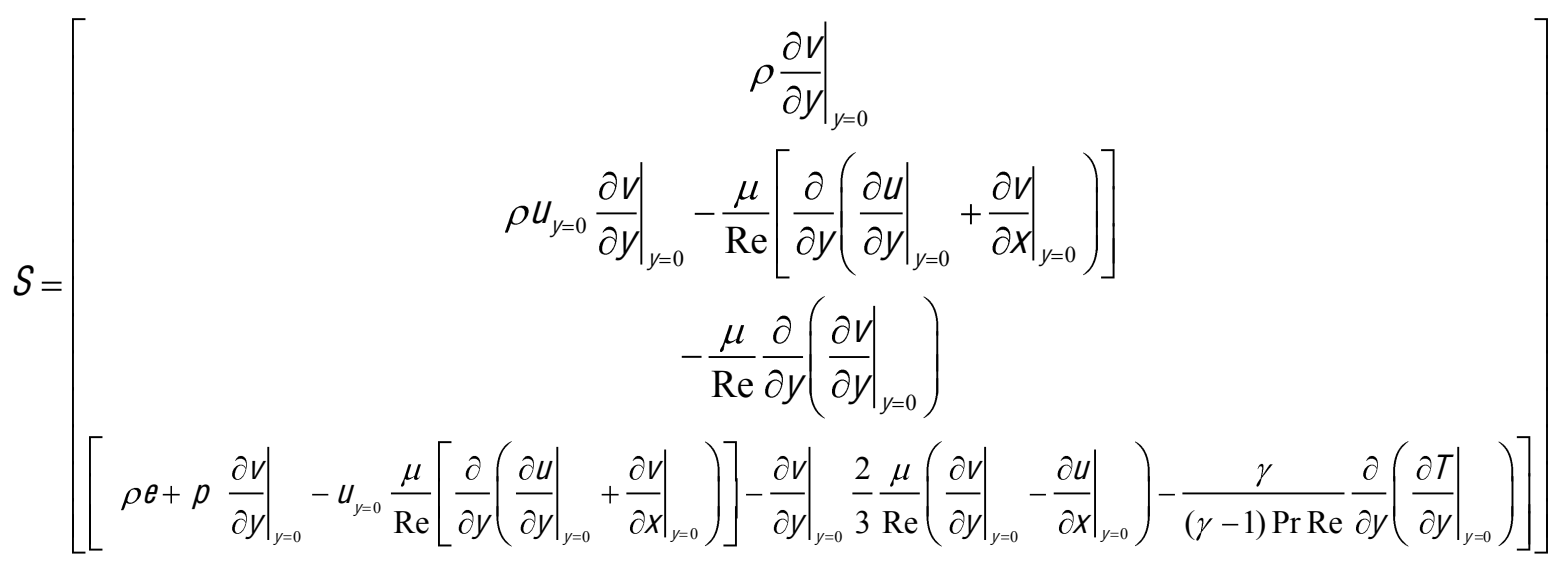

Applying same procedure to shear stresses and heat fluxes we get new term without singularity problem at $\mathrm{y}=0$.

$$
\begin{array}{ll}
\tau_{x x}=\frac{2}{3} \frac{\mu}{\operatorname{Re}}\left[\left.2\left(\frac{\partial u}{\partial x}-\frac{\partial v}{\partial y}\right)\right|_{y=0}\right] & \tau_{y y}=\left.\frac{2}{3} \frac{\mu}{\operatorname{Re}}\left(\frac{\partial v}{\partial y}-\frac{\partial u}{\partial x}\right)\right|_{y=0} \\
\tau_{\theta \theta}=\left.\frac{2}{3} \frac{\mu}{\operatorname{Re}}\left(\frac{\partial v}{\partial y}-\frac{\partial u}{\partial x}\right)\right|_{y=0} & \tau_{x y}=0 \\
q_{x}=-\left.\frac{\gamma}{(\gamma-1) \operatorname{Pr} \operatorname{Re}} \frac{\partial T}{\partial x}\right|_{y=0} & q_{y}=0
\end{array}
$$

Here $(x, y)$ are the two-dimensional and $(x, y, \theta)$ are the axisymmetric coordinates and $(u, v)$ are the corresponding velocity components, $\rho$ is the density, $p$ is the pressure. $E$ is the total energy given by 


$$
\begin{aligned}
& E=e+\frac{u^{2}+v^{2}}{2} \\
& e=c_{v} T, \quad p=\rho R T
\end{aligned}
$$

Here $e$ is the molecular internal energy and $T$ is the temperature.

The viscosity $(\mu)$ is computed using Sutherland's law and the coefficient of conductivity $(k)$ is given in terms of Prandtl number $(P r)$. The variables $\rho, p, T$ and velocity are non-dimensionalized by their corresponding reference variables $\rho_{\infty}, p_{\infty}, T_{\infty}$ and $\sqrt{R T}$ respectively. The reference value for length is computed by $\sqrt{\nu X_{0} / U_{\infty}}$, where $x_{0}$ is the location of the beginning of the computational domain in the stream wise direction. For the computation, the equations are transformed from physical coordinate system $(X, y)$ to the computational curvilinear coordinate system $(\xi, \eta)$ in a conservative manner and the governing equations become

$$
\frac{\partial \bar{Q}}{\partial t}+\frac{\partial \bar{F}}{\partial \xi}+\frac{\partial \bar{G}}{\partial \eta}=\frac{\partial \bar{F}_{v}}{\partial \xi}+\frac{\partial \bar{G}_{v}}{\partial \eta}+\bar{S}
$$

The components of the flux in the computational domain related to the flux in the physical domain by

$$
\bar{Q}=\frac{Q}{J}, \quad \bar{F}=\frac{J}{|J|} F, \quad \bar{G}=\frac{J}{|J|} G, \quad \bar{F}_{v}=\frac{J}{|J|} F_{v}, \quad \bar{G}_{v}=\frac{J}{|J|} G, \quad \bar{S}=\frac{J}{|J|} S \text { and } J=\left[\frac{\partial(\xi, \eta)}{\partial(x, y)}\right]
$$

\section{Solution Algorithm}

The governing equations are solved using $5^{\text {th }}$ order accurate weighted essentially non-oscillatory (WENO) scheme for space discretization and using $3^{\text {rd }}$ order total-variation-diminishing (TVD) Runge-Kutta scheme for time integration. These methods are suitable in flows with discontinuities or high gradient regions. These schemes solve the governing equations discretely in a uniform structured computational domain in which flow properties are known at the grid nodes. WENO scheme approximate the spatial derivatives in a given direction to a higher order at the nodes, using the neighboring nodal values in that direction. TVD-RK scheme integrates the resulting equations in time to get the point values as a function of time. Since the spatial derivatives are independent of the coordinate directions, the method can easily add other dimensions. It is well known that approximating a discontinuous function by a higher order (two or more) polynomial generally introduces oscillatory behavior near the discontinuity, and this oscillation increases with the order of the approximation. The essentially non oscillatory (ENO) and the improvement of these WENO methods are developed to keep the higher order approximations in the smooth regions and to eliminate or suppress the oscillatory behavior near the discontinuities. They are achieved by systematically adopting or selecting the stencils based on the smoothness of the function, which is being approximated. Shu ${ }^{36}$ explains the WENO and the TVD methods and the formulas. Atkins ${ }^{37}$ gives the application of ENO method to the N-S equations. Balakumar ${ }^{38}$ describes in detail the solution method implemented in this computation.

Four different types of boundary conditions are used in steady and unsteady calculations. At the outflow boundary, extrapolation boundary condition is used. At the wall, the simulation uses viscous conditions for the velocities and adiabatic or isothermal wall conditions, and it computes density from the continuity equation. In the mean flow computations, the simulation prescribes the free-stream values at the outer boundary, which lies outside the bow shock. The blunt cone is assumed to align with the free stream. In the unsteady computations, it superimposes the acoustic perturbations to the uniform mean flow at the upper boundary. The procedure is to first compute the steady mean flow by performing unsteady computations using a variable time step until the maximum residual reaches a small value $\sim 10^{-11}$. These computations use a CFL number of 0.2 for adiabatic case and 0.1 for isothermal cases. The next step is to introduce unsteady disturbances at the upper boundary of the computational domain and to perform time accurate computations to investigate the interaction and evolution of these disturbances to downstream. Since we use very fine spatial grid to resolve the leading edge region, these computations require very small time step which is taken as the minimum time step allowable for aforementioned CFL numbers. 
The grid is generated using analytical formulae. The grid stretches in the $\eta$ direction close to the wall and is uniform outside of the boundary layer. In the $\xi$ direction, the grid is symmetric about the leading edge and very fine near the nose and is uniform in the flat region. The outer boundary that lies outside the shock follows a parabola so that the boundary layer growth could be captured accurately. The computational domain extends from $x=-0.015$ to 20.0 inches in the axial direction in this computation depending on the wall temperature. Calculations were performed using a grid which has 32 blocks and each block has $127 \times 425$ grid points. Computational domain has approximately 2 million grid points. Due to the very fine grid requirement near the nose, the allowable time step is very small and the computations become very expensive to simulate the unsteady computations in the entire domain.

The acoustic field that impinges on the outer boundary is taken to be in the following form.

$$
p^{\prime}=\operatorname{Real}\left\{\hat{p} e^{j \alpha_{a c} x \pm \varepsilon_{a c} y-i \omega t}\right\}
$$

Here $\alpha_{a c}, \varepsilon_{a c}$ are the $x, y$ wave numbers, respectively, of the acoustic wave and $\omega$ is the corresponding frequency of the acoustic disturbance. The incident angle $\theta$ of the acoustic wave is defined as

$$
\theta=\tan ^{-1} \frac{\varepsilon_{a c}}{\alpha_{a c}}
$$

and in this study computations are performed for zero incidence angles.

\section{Results and Discussion}

Computations are performed for hypersonic flow at a free stream Mach number of 6.0 over a 5-degree half-angle cone with blunt leading edge, $\mathrm{R}_{\mathrm{n}}=0.001$ in, for different wall temperatures to investigate the effects of wall cooling on hypersonic boundary layer receptivity due to acoustic disturbances in slow mode. The flow parameters are given in Table 1 and the boundary layer edge conditions for a sharp cone are given in Table 2. Figure 1 shows the schematic diagram of the computational set up. The nose region of the cone is modeled as a circle. Simulations are performed for wall temperatures $\mathrm{T}_{\mathrm{w}}=$ Adiabatic wall $\left(\mathrm{T}_{\mathrm{aw}}\right), 0.75 * \mathrm{~T}_{\mathrm{aw}}, 0.50 * \mathrm{~T}_{\mathrm{aw}}, 0.40 * \mathrm{~T}_{\mathrm{aw}}, 0.30 * \mathrm{~T}_{\mathrm{aw}}$, and $0.20 * \mathrm{~T}_{\mathrm{aw}}$. Different cases are summarized in Table 3.

Table 1. Flow parameters for the wind tunnel model (Horvath et. al. ${ }^{39}$ )

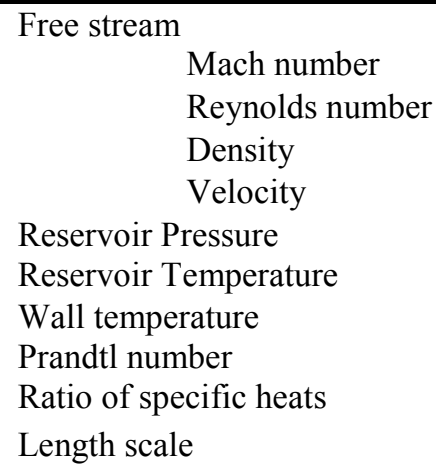

$$
\begin{aligned}
\mathrm{M}_{\infty} & =6.0 \\
\mathrm{Re}_{\infty} & =7.8 \times 10^{6} / \mathrm{ft} \\
\rho_{\infty} & =7.059 \times 10^{-3} \mathrm{lbm} / \mathrm{ft}^{3} \\
\mathrm{U}_{\infty} & =3140.21 \mathrm{ft} / \mathrm{s} \\
\mathrm{P}_{0} & =475 \mathrm{psi} \\
\mathrm{T}_{0} & =475^{\circ} \mathrm{F}
\end{aligned}
$$

Adiabatic and Isothermal conditions

$\operatorname{Pr}=0.72$

$\gamma=1.4$

$\sqrt{v_{\infty} X_{0} / U_{\infty}}=7.308 \times 10^{-3} \mathrm{ft}$

Table 2. Parameters along the cone surface.

\begin{tabular}{lc}
\hline (Var.) $)_{\text {edge }} /(\text { Var. })_{\infty}$ & Sharp Cone \\
\hline Mach Number & 0.932 \\
Pressure Ratio & 1.560 \\
Density Ratio & 1.372 \\
Temperature Ratio & $1.137^{*}$ \\
\hline
\end{tabular}

*Adiabatic case. 
Table 3. Wall temperatures and bow shock standing distances.

\begin{tabular}{cccc}
\hline$T_{\text {wall }} / T_{\text {Ad,wall }}$ & $G_{0}=T_{\text {wall }} / T_{\infty}$ & $T_{\text {wall }}\left({ }^{\circ} \mathrm{R}\right)$ & $\delta\left(\times 10^{-4}\right.$ in. $)$ \\
\hline 1.00 & 7.052 & 804 & 2.50 \\
0.75 & 5.289 & 603 & 2.39 \\
0.50 & 3.526 & 402 & 2.28 \\
0.40 & 2.8208 & 322 & 2.21 \\
0.30 & 2.1156 & 241 & 2.15 \\
0.20 & 1.4104 & 161 & 2.13 \\
\hline
\end{tabular}

$T_{\infty}=113.98^{\circ} \mathrm{R}$.

\section{Linear Stability}

Linear stability computations done for flow over an axisymmetric cone at a free stream Mach number of $\mathrm{M}=6$ at different wall temperature conditions $T_{w} / T_{a w}=1.0,0.50$ and 0.20 . Figures 2(a), (b) and (c) depict the results in (Re, $F),(R e, \alpha)$ and $\left(R e, C_{r}\right)$ planes for two-dimensional disturbances respectively. It is seen that with the wall cooling (1) the first mode is stabilized (2) the unstable frequencies increase (3) the unstable wave number increases and (4) the range of the phase speeds of the unstable waves narrows.

Figure 3(a)-(d) show the N-Factor curves for decreasing frequencies at different wall temperature conditions. Most amplified frequencies for wall temperatures $T_{w} / T_{a w}=1.0,0.75,0.50$ and 0.20 are found as $0.8 \times 10^{4}, 0.9 \times 10^{4}$, $1.1 \times 10^{4}$, and $1.7 \times 10^{4}$ for $N=10$. Here the variables are non-dimensionalized by the variables at the edge of boundary layer. To obtain the variables non-dimensionalized by the free stream values as given in Table 1 , the variables in this section should be multiplied by the appropriate factors from Table 2 . The frequency variable $F$ has to be multiplied by 1.174 to obtain the values in terms of free stream values.

The neutral stability curve clearly shows the unstable first and the second mode regions for adiabatic wall temperature but when cooling applied the unstable first mode region disappears. The first mode and the second mode neutral stability curves merge at a Reynolds number of $R e=1600$ for the cone.

\section{Mean Flow}

Validation studies of axisymmetric code presented in a previous study ${ }^{33}$. Figure 4 shows the mean flow temperature contours computed using the WENO code. The figures 5(a)-(d) show the results for the 5 -degree halfangle cone at different wall temperatures $T_{w}=$ Adiabatic wall $\left(T_{a w}\right), 0.75^{\star} T_{a w}, 0.50^{\star} T_{a w}$ and $0.20^{\star} T_{a w}$. In the adiabatic wall case bow shock generated a high temperature region. This region convected to downstream over cone wall. For cooled wall cases the high temperature region is trapped between the bow shock and nose part of the cone. Bow shock standing distance reduced $\% 15$ when wall temperature reduced to $0.20^{\star} T_{a w}$ (Table 3). Figure 5(a) and 5 (b) depict the density contours over adiabatic cone and cooled cone $\left(T_{w}=0.20^{\star} T_{a w}\right)$ cases. Maximum nondimensional density occurred on the stagnation point for adiabatic and cooled conditions are 6 and 33 respectively. Over the flat part of the cone density contours looks similar. High density region is increased when cooling applied but it did not extend to flat part. Figure 6 compares the Mach contours of the aforementioned wall conditions. Wall cooling decreased the oblique shock angle and compressed the Mach layers to wall. Figure 7(a) shows the wall to free stream temperature ratio, $G_{0}$, along the cone surface for different wall cooling conditions and Fig. 7(b) shows the variation of boundary layer edge Mach number, $M_{\theta}$, along cone surface. Edge Mach number and temperature for adiabatic case at $X=13.96 \mathrm{in}$. are $M_{e}=5.575$ and $T_{e}=129.53{ }^{\circ} \mathrm{R}$ and for $80 \%$ cooled case at $X=19.58 \mathrm{in}$. are $M_{e}=5.581$ and $T_{e}=129.26^{\circ} \mathrm{R}$.

Density profiles at different $x$ locations are plotted in Figures 8(a)-(d) in similarity coordinates for adiabatic wall and cooled walls $\left(0.50^{\star} T_{a w}, 0.30^{\star} T_{a w}\right.$, and $\left.0.20^{\star} T_{a w}\right)$. At $x=0.2 \mathrm{in}$. density profiles converges to a profile which stays the same until the end of the computational domain. Wall cooling decreased boundary layer thickness from $\eta=14.6\left(T_{a w}\right)$ to $\eta=8.19\left(0.20^{\star} T_{a w}\right)$ and also increased the density on wall from $0.16\left(T_{a w}\right)$ to $0.80\left(0.20^{\star} T_{a w}\right)$. Density increase on the wall changed the characteristic of density profiles. In adiabatic case minimum density occurred on cone wall and steadily increased away from it. However, for cooled wall case $\left(T_{w}=0.20^{\star} T_{a w}\right)$ density on the wall $\left(\rho_{\eta=0}=0.80\right)$ first started to decrease away from the wall until $\eta=2\left(\rho_{\eta=2}=0.42\right)$. After making a minimum 
at this point density changed its character and increased as in the adiabatic wall case. Even this density profiles seems to have a low density region their minimum density at $\eta=2\left(\rho_{\eta=2}=0.42\right)$ is 2.6 times larger than the minimum of adiabatic case $\left(\rho_{\eta=0}=0.16\right)$. As a result wall cooling reduced the boundary layer thickness and increased the density inside it.

Figures 9(a)-(f) show the temperature profiles at different axial locations for different cooling cases. The compressible Blasius similarity profiles are also included for comparison. In adiabatic wall case at $x=0.2 \mathrm{in}$. calculated temperature profiles perfectly matched with similarity solution (Fig. 9(a)). As was observed in the density graphs, temperature profiles are also converged to the same profile at $x=0.2 \mathrm{in}$. and stayed same until the end of the computational domain. Wall cooling decreased thermal boundary layer thickness also. For highest cooling case $\left(T_{w}=0.20^{\star} T_{a w}\right)$ wall temperature was $161^{\circ} R$ and it increased away from wall until $\eta=2\left(T_{\eta=2}=305^{\circ} R\right)$. After making a maximum at this point temperature of the flow decreased as in the adiabatic case to $129.26^{\circ} R$. The difference between the similarity profiles and simulation results comes from the nose bluntness, $R_{n}=0.001$ in. Figure 10 (a) and (b) show the mean flow density profiles at different axial locations in physical coordinates for adiabatic $\left(804^{\circ} R\right)$ and highest wall cooling case $\left(161^{\circ} R\right)$ respectively. Minimum density observed at $y=0.01 \mathrm{in}$. normal to wall. Figure 11(a) and (b) depicts the mean flow temperature profiles at different axial locations in physical coordinates for adiabatic $\left(804^{\circ} R\right)$ and cooled wall $\left(161^{\circ} R\right)$ conditions.

\section{Interaction of Slow Acoustic Waves with the Boundary Layer}

After the mean flow is computed two dimensional slow acoustic disturbances are introduced at the outer computational boundary and the time accurate simulations are performed. Unsteady simulation results are presented for the cases $T_{w}=T_{a w}, 0.75^{\star} T_{a w}, 0.50^{\star} T_{a w}$, and $0.20^{\star} T_{a w}$ (Slow and Fast waves) at the most amplified frequencies $0.96 \times 10^{4}, 1.08 \times 10^{4}, 1.32 \times 10^{-4}$, and $2.0 \times 10^{4}$. These frequencies correspond to $375,421,515$, and $780 \mathrm{kHz}$ respectively. These frequencies give maximum amplification within the computational domain. To remain in the linear regime, the amplitude of the forcing freestream acoustic waves is given a small value of $2 \times 10^{5}$ for the first three cases and $2 \times 10^{6}$ for $0.20^{\star} T_{a w}$ case. Even with these small amplitudes, nonlinearity starts to develop near the end of computational domain for adiabatic case.

Figures 12(a)-(e) show the evolution of the wall pressure fluctuations for aforementioned cases in linear scale while Figures 13(a)-(e) depict the results in a log scale. The figures clearly show the generation and the eventual exponential growth of the instability waves inside the boundary layer for adiabatic and cooled wall conditions. For the adiabatic wall conditions, Fig. 12(a) and Fig. 13(a), the disturbances grow from the leading edge and reach large amplitude levels of 0.50 near the predicted transition onset point at $X=19 \mathrm{in}$. The slow wave whose wavelength is closer to the wavelength of the instability wave transforms into instability wave smoothly near the nose region. The parallel linear computations show that the first mode amplifies starting from leading edge weakly up to $x \sim 15$ inch for this frequency.

Figure 13(b) shows the pressure fluctuations for $T_{w}=0.75^{\star} T_{a w}$ in log scale. It is evident that wall cooling reduced the growth of the first mode until $x=6$ in. then growth of the second mode is observed. Because of wall cooling maximum amplitude only reached to 0.734 which is in the same order of adiabatic case. When more wall cooling $\left(T_{w}=0.50^{\star} T_{a w}\right)$ is applied stabilization of the first mode become obvious. In Figure 13(c) the neutral point appeared at $X=5.55 \mathrm{in}$. with an amplitude of $6.18 \times 10^{6}$. Maximum amplitude for this case is 0.041 at $X=10.73 \mathrm{in}$. The neutral points are not discernable for adiabatic and $T_{w}=0.75^{\star} T_{a w}$ but it appeared for $T_{w}=0.50^{\star} T_{a w}$ and stayed in the picture for cooler cases.

Figure 13(d) and (e) show the wall pressure fluctuations for slow and fast waves respectively at $T_{w}=0.20^{\star} T_{a w}$. In these figures the neutral points are located at $4.94 \mathrm{in}$. (Slow Wave) and $5.62 \mathrm{in}$. (Fast Wave) with the amplitudes of $2 \times 10^{8}$ and $1 \times 10^{6}$ respectively. Maximum wall pressure amplitudes 0.00023 for slow wave and 0.0029 for fast wave are observed at $x=7.6$ in for both cases. Because of the difference in the initial amplitude of the acoustic disturbances the maximum amplitudes for adiabatic wall, $0.75{ }^{\star} T_{a w}$ and $0.50{ }^{\star} T_{a w}$ need to be divided by 10 and they become 0.05 , 0.0734 , and 0.0041 respectively. While the maximum amplitude of $0.2{ }^{\star} T_{a w}$ (Slow Wave) simulation, 0.00023, is almost 20 times less than $0.50{ }^{\star} T_{a w}$ simulation, maximum amplitude of $0.20{ }^{\star} T_{a w}$ (Fast Wave) simulation is on the same order.

Also it is interesting to observe that the first mode is not stabilized for fast wave case in Figure 13(e) while Figures 13(a)-(d) clearly show the stabilization effect of wall cooling on the first mode for slow wave cases. 
Simulated transition locations for $T_{w}=T_{a w}, 0.75^{\star} T_{a w}, 0.50^{\star} T_{a w}$, and $0.20^{\star} T_{a w}$ (Slow and Fast waves) wall temperature conditions are $19 \mathrm{in}, 13 \mathrm{in}, 10.7 \mathrm{in}$, and $7.6 \mathrm{in}$. respectively. From these results one can conclude that wall cooling is destabilizing the boundary layer and transition points are moving to upstream.

Another set of simulations run for $T_{w}=T_{a w}, 0.75^{\star} T_{a w}, 0.50^{\star} T_{a w}, 0.40^{\star} T_{a w}, 0.30^{\star} T_{a w}$, and $0.20^{\star} T_{a w}$ using the same forcing disturbance frequency, $F=1.2 \times 10^{4}$, in slow wave mode to observe the effects of wall cooling on development of instability waves in the same disturbance environment. Figure 14 and 15 shows the unsteady wall pressure fluctuations for aforementioned simulations.

Figure 15(b) shows the pressure fluctuations for $T_{w}=0.50^{\star} T_{a w}$ in log scale. It is evident that wall cooling reduced the growth of first mode until $x=6.96$ in where the amplitude is $6.5 \times 10^{6}$ then growth of second mode observed. Because of the wall cooling maximum amplitude only reached to 0.0035 which is 35 times less than of the adiabatic case. Stabilization of the first mode become apparent with wall cooling as was seen in neutral stability diagrams (Figure 2(a)). Figure 15(c) shows the exponential decrease of amplitude of wall pressure fluctuations for $T_{w}=0.40^{\star} T_{a w}$ simulation until the neutral point located at $x=8.46 \mathrm{in}$. with an amplitude of $9.95 \times 10^{7}$. Maximum amplitude for this case is 0.129 occurred at $x=14.62 \mathrm{in}$. More cooling increased the maximum amplitude level to the same value of adiabatic case. It clearly shows the destabilization effect of wall cooling on second mode. Finally for $T_{W}=0.20^{\star} T_{a w}$ simulation the maximum amplitude increased more than two times and reached to 0.301 at the end of the computational domain $x=19$.9in. Further cooling moved the neutral point to $x=13.4$ in where the amplitude is $4.7 \times 10^{8}$. In these simulations neutral point was not discernable for adiabatic wall and $T_{w}=0.75^{\star} T_{a w}$ but it appeared for $T_{w}=0.50^{\star} T_{a w}$ and cooler wall temperatures because of the stabilization of the first mode (Slow Wave) with wall cooling. Transition locations computed for $T_{w}=T_{a w}, 0.50{ }^{\star} T_{a w}, 0.40^{\star} T_{a w}$ and $0.20^{\star} T_{a w}$ wall temperature conditions for slow wave acoustic disturbances are 9.47in, 9.67in, 14.62in, and 19.9in. respectively. Transition location is delayed with wall cooling for the same slow wave frequency because of the stabilization of first mode. However the amplitudes of wall pressure fluctuations increased with wall cooling to larger values than of adiabatic case. The delay of the transition locations and comparison of the amplitude levels can be easily seen in Figure 16.Figure 17 shows the variation of eigenvalues $\left(\mathrm{r}, \alpha_{\mathrm{i}}\right)$ for the slow and fast waves for $F=1.2 \times 10^{4}$ at $T_{w}=0.20^{\star} T_{\text {aw }}$.

Figure 18 compares the evolution of unsteady density fluctuations obtained from the simulations for slow wave $\left(F=1.2 \times 10^{-4}\right)$ at a fixed time for adiabatic and cooled wall $\left(T_{w}=0.20^{\star} T_{a w}\right)$ conditions. Figure 18 (a) and (b) clearly show that there is no entropy layer generated for small bluntness case and the disturbances excite the boundary layer up to the wall. These figures clearly show the disturbance evolution in the nose region. Acoustic disturbances pass the bow shock and directly enter the boundary layer. Figure 16 (b) shows that the boundary layer is thinner and amplitude of the fluctuations inside the boundary layer is weaker for cooled wall compared to adiabatic wall presented in Figure 18(a).

Figure 19 and 20 shows the propagation of density fluctuations inside boundary layer from leading edge to the end of the computational domain. In Figure 19(a) disturbances interact with the bow shock in the nose region and directly enter to the boundary layer. Disturbances form rope shape structure inside the boundary layer and on the oblique shock. The interaction is obvious between the density fluctuations on the oblique shock and boundary layer. Figure 19 (b) shows the disturbance field from 0.3in. to $2.0 \mathrm{in}$. In this figure four different zones are observed similar to previous studies ${ }^{6,11-12}$. First zone is the area outside the shock where acoustic disturbances propagate uniformly. In the second zone acoustic waves transmitted through shock layer. The third zone is the area between the shock and the boundary layer. This region consists of transmitted external acoustic field and the disturbances radiated from the boundary layer. It is interesting to see that the third region is much quieter compared to the acoustic waves outside the shock layer. This implies that the acoustic waves are weakly transmitted through the shock. The fourth zone is the boundary layer where the boundary layer disturbances evolve. Figure 19(c) clearly shows decay of density fluctuation amplitudes from order of $10^{5}$ to $10^{8}$ which corresponds to the first mode stabilization. In Figure 20(a) and (b) density fluctuations inside the boundary layer are not discernable and the region between shock layer and cone wall seems quiet. However, acoustic disturbances propagated from $4 \mathrm{in}$. to 16 in. in the order of $10^{7}$ or below then started to gain amplitude and became visible (also look Fig. 15 (d)). This phenomenon shows the necessity of high resolution grid and higher order accurate schemes. Finally Figure 20 (c) shows destabilization of the second mode disturbances near the end of the computational domain.

\section{Discussion and Conclusion}

The receptivity and the stability of hypersonic boundary layers over a blunt cone with 5 degrees half-angle with nose radius $0.001 \mathrm{in}$. are numerically investigated at a free stream Mach number of 6.0 and at a Reynolds number of 
$7.8 \times 10^{6} / \mathrm{ft}$. Both steady and unsteady solutions are obtained by solving compressible Navier-Stokes equations using the $5^{\text {th }}$ order accurate weighted essentially non-oscillatory (WENO) scheme for space discretization and using a $3^{\text {rd }}$ order total-variation-diminishing (TVD) Runge-Kutta scheme for time integration. Unsteady flow is forced using slow and fast acoustic disturbance waves with the most amplified disturbances calculated based on mean flow analyses and also forced with non-dimensional frequency of $F=1.2 \times 10^{-4}$. Computations are performed for different wall temperatures 804 (adiabatic wall), 603, 402, 322, 241, and $161^{\circ} \mathrm{R}$.

Table 4 summarizes computation parameters and the receptivity coefficients for the most amplified slow and fast mode acoustic disturbances at different wall temperature conditions. It is shown that the first mode of slow wave acoustic disturbances stabilized by wall cooling. However, wall cooling also caused the destabilization of the second mode and transition location moved to upstream from $x=19.1$ in for adiabatic case to $x=7.61$ in highly cooled wall case. It is also interesting to observe that wall cooling did not affect the first mode of fast acoustic disturbance waves. The receptivity coefficient of the fast wave case is 50 times greater than of slow wave case. We can conclude that the boundary layer is much more receptive to fast acoustic waves as compared to the slow wave.

Table 4. Computation parameters and receptivity coefficients for the most amplified frequencies at different wall temperatures.

\begin{tabular}{|c|c|c|c|c|c|}
\hline$F \times 10^{-4}$ & $T_{\text {wall }} / T_{a w}$ & $\left(P_{\text {wall }}\right)_{n p}$ & $C_{\text {recpt. }}=\left(P_{\text {wall }}\right)_{n p} / P_{a c}$ & $x_{T r .}(i n)$. & $\left(P_{\text {wall }}\right)_{T r .}$ \\
\hline $0.96^{S}$ & 1.00 & $8.5 \times 10^{-6}$ & $4.23 * *$,\# & 19.1 & 0.552 \\
\hline $1.08^{S}$ & 0.75 & $4.8 \times 10^{-5}$ & $2.39^{*}, \#$ & 13.1 & $0.0734^{+}$ \\
\hline $1.32^{\mathrm{S}}$ & 0.50 & $6.2 \times 10^{-6}$ & $0.309 *$ & 10.7 & $0.0041^{+}$ \\
\hline $2.0^{S}$ & 0.20 & $2.0 \times 10^{-8}$ & $0.010 * *$ & 7.61 & 0.0002 \\
\hline $2.0^{F}$ & 0.20 & $1.0 \times 10^{-6}$ & $0.500 * *$ & 7.59 & 0.0029 \\
\hline
\end{tabular}

The transition locations stayed almost same for adiabatic wall and cooled walls $\left(603^{\circ} R\right.$ and $\left.402^{\circ} R\right)$ respectively for $9.475 \mathrm{in}$., $9.714 \mathrm{in}$. and $9.672 \mathrm{in}$. Transition locations increased dramatically for wall temperatures $322^{\circ} \mathrm{R}, 241^{\circ} \mathrm{R}$ and $161^{\circ} \mathrm{R}$ to $14.628,17.013$, and 19.906 inches. This is happened due to the stabilization of the first mode disturbances. However, amplitude of wall pressure fluctuations of cooled wall case $\left(T_{w}=161^{\circ} R\right)$ increased 2.34 times of the adiabatic wall case $\left(T_{w}=T_{a w}=804^{\circ} \mathrm{R}\right)$. Table 5 summarizes the simulation parameters and gives the receptivity coefficients for the same slow wave disturbance frequency, $F=1.2 \times 10^{4}$, at different wall temperature conditions.

Table 5. Computation parameters and receptivity coefficients for the same slow wave disturbance frequency at different wall temperatures.

\begin{tabular}{cccccc}
\hline$T_{\text {wall }}\left({ }^{\circ} \mathrm{R}\right)$ & $T_{\text {wall }} / T_{\text {aw }}$ & $x_{\text {np. }}($ in. $)$ & $C_{\text {recpt. }}={ }^{\left(P_{\text {wall }}\right)_{n p} / P_{a c}}$ & $x_{\text {Tr. }}$ (in. $)$ & $\left(P_{\text {wall }}\right)_{\text {Tr. }}$ \\
\hline 804 & 1.00 & 0.207 & $1.5225^{\#}$ & 9.475 & 0.129 \\
603 & 0.75 & 0.207 & $1.4613^{\#}$ & 9.714 & 0.046 \\
402 & 0.50 & 6.965 & 0.3246 & 9.672 & 0.003 \\
322 & 0.40 & 8.466 & 0.0497 & 14.628 & 0.129 \\
241 & 0.30 & 10.964 & 0.0059 & 17.013 & 0.068 \\
161 & 0.20 & 13.397 & 0.0023 & 19.906 & 0.301 \\
\hline
\end{tabular}

$T_{\infty}=113.98^{\circ} R, P_{a c}=2 \times 10^{-5}$, Nose Radius $R_{n}=0.001$ in. ${ }^{\#}$ Neutral point is not observed for this case and amplitude from $x=0.207$ is used.

Because of the initial growth of first mode in adiabatic and cooled wall $\left(T_{w}=0.75^{\star} T_{a w}\right)$ neutral points are not observed. Therefore wall pressure fluctuations at $0.207 \mathrm{in}$. are used in the receptivity coefficient calculations. Neutral point locations moved downstream with wall cooling. Receptivity coefficients are 1.5225, 1.4613, 0.3246, $0.0497,0.0059$, and 0.0023 respectively for wall temperatures $804,603,402,322,241$, and $161{ }^{\circ} \mathrm{R}$. 
Wall cooling reduced receptivity coefficients and increased the transition Reynolds numbers. The receptivity coefficient for adiabatic wall case is 1.5225 and they are much smaller in the order of $10^{-3}$ for highly cooled cones $\left(241\right.$, and $\left.161^{\circ} \mathrm{R}\right)$. This raises some questions ${ }^{34}$ about the transition process over cone with small bluntness. If the receptivity coefficients are very small for the second modes as was found in this paper, how can the amplitude of the disturbances reach such high values? Most probably non-zero acoustic incident angles may produce larger receptivity coefficients than zero incident angle used in this paper.

\section{Acknowledgement}

The first and the third authors are supported by Flow Physics and Control Branch of NASA Langley Research Center for this work.

\section{References}

${ }^{1}$ Lees, L., "The Stability of the Laminar Boundary Layer in a Compressible Fluid," NACA Report 876, 1947.

${ }^{2}$ Mack, L.M., "Boundary-Layer Stability Theory," AGARD-R-709, 1984.

${ }^{3}$ Demetriades, A., "New Experiments on Hypersonic Boundary Layer Stability Including Wall Temperature Effects," Proceedings of the Heat Transfer and Fluid Mechanics Institute, Vol. 26, 1978, pp. 39-54.

${ }^{4}$ Lysenko, V.I., Maslov, A.A. and Semenov, N.M., "Experimental Study of the Effect of Wall Heating on the Transition and Stability of Supersonic Boundary Layers," Fluid Mechanics - Soviet Research, Vol. 11 (6), 1982, pp. 32-43.

${ }^{5}$ Stetson, K.F., Thompson, E.R., Donaldson, J.C. and Siler, L.G., "Laminar Boundary Layer Stability Experiments on a Cone at Mach 8. Part 5 - Test with a Cooled Model," 20th Fluid Dynamics, Plasma Dynamics and Lasers Conference, Buffalo, NY, AIAA 1989-1895.

${ }^{6}$ Balakumar, P. and Malik, M.R., "Effects of Adverse Pressure Gradient and Wall Cooling Instability of Hypersonic Boundary Layers," HTC-9404, 1994.

${ }^{7}$ Morkovin, M.V., "Critical Evaluation of Transition from Laminar to Turbulent Shear Layers with Emphasis on Hypersonically Travelling Bodies," AFFDL-TR-68-148, 1969.

${ }^{8}$ Reshotko, E., "Boundary Layer Stability and Transition," Annu. Rev. Fluid Mech., Vol. 8, 1976, pp. 311-349.

${ }^{9}$ Maslov, A.A., Shiplyuk, A.N., Sidorenko, A.A. and Arnal, D., "Leading-Edge Receptivity of a Hypersonic Boundary Layer on a Flat Plate," Journal of Fluid Mechanics, Vol. 426, 2001, pp. 73-94.

${ }^{10}$ Maslov, A.A., Shiplyuk, A.N., Bountin, D.A. and Sidorenko, A.A., "Mach 6 Boundary-Layer Stability Experiments on Sharp and Blunted Cones," Journal of Spacecrafts and Rockets, Vol. 43 (1), 2006, pp. 71-76.

${ }^{11}$ Borg, M.P., Schneider, S.P. and Juliano, T.J., "Effect of Freestream Noise in Roughness-Induced Transition for the X-51A Forebody," 46th Aerospace and Sciences Meeting \& Exhibit, Reno, NV, AIAA 2008-0592.

${ }^{12}$ Rufer, S. and Schneider, S.P., "Hot-Wire Measurements of Stability Waves on Cones at Mach 6," 36th AIAA Fluid Dynamics Conference and Exhibit, San Francisco, CA, AIAA 2006-3054.

${ }^{13}$ Rufer, S. and Schneider, S.P., "Hot-Wire Measurements of Instability Waves on a Blunt Cone at Mach 6," 35th AIAA Fluid Dynamics Conference, Toronto, Canada, AIAA 2005-5137.

${ }^{14}$ Fedorov, A.V., "Prehistory of Instability in a Hypersonic Boundary Layer," Theoret. Comput. Fluid Dynamics, Vol. 14, 2001, pp. 359-375.

${ }^{15}$ Fedorov, A.V., Malmuth, N.D., Rasheed, A. and Hornung, H.G., "Stabilization of Hypersonic Boundary Layers by Porous Coatings," AIAA Journal, Vol. 39, 2001, pp. 605.

${ }^{16}$ Fedorov, A.V., "Receptivity of Hypersonic Boundary Layer to Wall Disturbances," Theoret. Comput. Fluid Dynamics, Vol. 15, 2002, pp. 231-254.

${ }^{17}$ Fedorov, A.V., "Receptivity of a High-Speed Boundary Layer to Acoustic Disturbances," Journal of Fluid Mechanics, Vol. 491, 2003, pp. 101-129.

${ }^{18}$ Fedorov, A.V., Shiplyuk, A.N., Maslov, A.A., Burov, E.V. and Malmuth, N.D., "Stabilization of a Hypersonic Boundary Layer Using an Ultrasonically Absorptive Coating," Journal of Fluid Mechanics, Vol. 479, 2003, pp. 99-124.

${ }^{19}$ Tumin, A., "Receptivity of Compressible Boundary Layers to Three Dimensional Wall Perturbations," 44th Aerospace Sciences Meeting \& Exhibit, Reno, NV, AIAA-2006-1110.

${ }^{20}$ Collis, S.S. and Lele, S.K., "Receptivity to Surface Roughness Near a Swept Leading Edge," Journal of Fluid Mechanics, Vol. 380, 1999, pp. 141-168.

${ }^{21}$ Zhong, X., "Leading-Edge Receptivity to Freestream Disturbance Waves for Hypersonic Flow over Parabola," Journal of Fluid Mechanics, Vol. 441, 2001, pp. 315-367.

${ }^{22} \mathrm{Ma}$, Y. and Zhong, X., "Receptivity of a Supersonic Boundary Layer over a Flat Plate. Part 1. Wave Structures and Interactions," Journal of Fluid Mechanics, Vol. 488, 2003, pp. 31-78.

${ }^{23} \mathrm{Ma}$, Y. and Zhong, X., "Receptivity of a Supersonic Boundary Layer over a Flat Plate. Part 2. Receptivity to Freestream Sound," Journal of Fluid Mechanics, Vol. 488, 2003, pp. 79-121.

${ }^{24}$ Ma, Y. and Zhong, X., "Receptivity of a Supersonic Boundary Layer over a Flat Plate. Part 3. Effects of Different Types of Freestream Disturbances," Journal of Fluid Mechanics, Vol. 532, 2005, pp. 63-109. 
${ }^{25}$ Zhong, X., "Receptivity and Linear Stability of Stetson's Mach 8 Blunt Cone Stability Experiments," 32nd AIAA Fluid Dynamics Conference and Exibit, St. Louis, Missouri, AIAA 2002-2849.

${ }^{26}$ Zhong, X., "Receptivity of Mach 6 Flow over a Flared Cone to Freestream Disturbance," 42nd Aerospace Sciences Meeting and Exhibit, Reno, NV, AIAA 2004-0253.

${ }^{27}$ Zhong, X., "Effects of Nose Bluntness on Hypersonic Boundary Layer Receptivity over a Blunt Cone," 35th AIAA Fluid Dynamics Conference and Exhibit, Toronto, Canada, AIAA 2005-5022.

${ }^{28}$ Malik, M.R. and Balakumar, P., "Acoustic Receptivity of Mach 4.5 Boundary Layer with Leading-Edge Bluntness," Theoret. Comput. Fluid Dynamics, Vol. 21 (5), 2007, pp. 323-342.

${ }^{29}$ Malik, M.R. and Balakumar, P., "Receptivity of Supersonic Boundary Layers to Acoustic Disturbances " 35th AIAA Fluid Dynamics Conference and Exhibit, Toronto, Canada, AIAA-2005-5027.

${ }^{30}$ Balakumar, P., "Stability of Supersonic Boundary Layers Over Blunt Wedges," 36th AIAA Fluid Dynamics Conference and Exhibit, San Francisco, Ca, AIAA-2006-3053.

${ }^{31}$ Balakumar, P., "Control of Supersonic Boundary Layers Using Steady Suction," 36th AIAA Fluid Dynamics Conference and Exhibit, San Francisco, Ca, AIAA-2006-3058.

${ }^{32}$ Balakumar, P., "Receptivity of Supersonic Boundary Layers To Acoustic Disturbances Over Blunt Cones," 37th AIAA Fluid Dynamics Conference and Exhibit, Miami, FL, AIAA-2007-4491.

${ }^{33}$ Kara, K., Balakumar, P. and Kandil, O.A., "Receptivity of Hypersonic Boundary Layers Due to Acoustic Disturbances over Blunt Cone," 45th Aerospace Sciences Meeting and Exhibit, Reno, NV, AIAA 2007-0945.

${ }^{34}$ Kara, K., Balakumar, P. and Kandil, O.A., "Effects of Nose Bluntness on Stability of Hypersonic Boundary Layers over a Blunt Cone," 37th Fluid Dynamics Conference \& Exhibit, Miami, FL, AIAA 2007-4492.

${ }^{35}$ Egorov, I.V., Fedorov, A.V. and Soudakov, V.G., "Receptivity of a Hypersonic Boundary Layer over a Flat Plate with a Porous Coating," Journal of Fluid Mechanics, Vol. 601, 2008, pp. 165-187.

${ }^{36}$ Shu, C.-W., "Essentially Non-Oscillatory and Weighted Essentially Non-Oscillatory Schemes for Hyperbolic Conservation Laws," NASA CR-97-206253, 1997.

${ }^{37}$ Atkins, H.L., "High-Order ENO Methods for The Unsteady Compressible Navier-Stokes Equations," 10th AIAA Computational Fluid Dynamics Conference, Honolulu, HI, AIAA-1991-1557.

${ }^{38}$ Balakumar, P., Zhao, H. and Atkins, H.L., "Stability of Hypersonic Boundary Layers over a Compression Corner," 42nd AIAA Fluid Dynamics Conference and Exhibit, St. Louis, Missouri, AIAA-2002-2848.

${ }^{39}$ Horvath, T.J., Berry, S.A., Hollis, B.R., Chang, C.-L. and Singer, B.A., "Boundary Layer Transition on Slender Cones in Conventional and Low Disturbance Mach 6 Wind Tunnels," 32nd AIAA Fluid Dynamics Conference, St. Louis, Missouri, AIAA 2002-2743. 


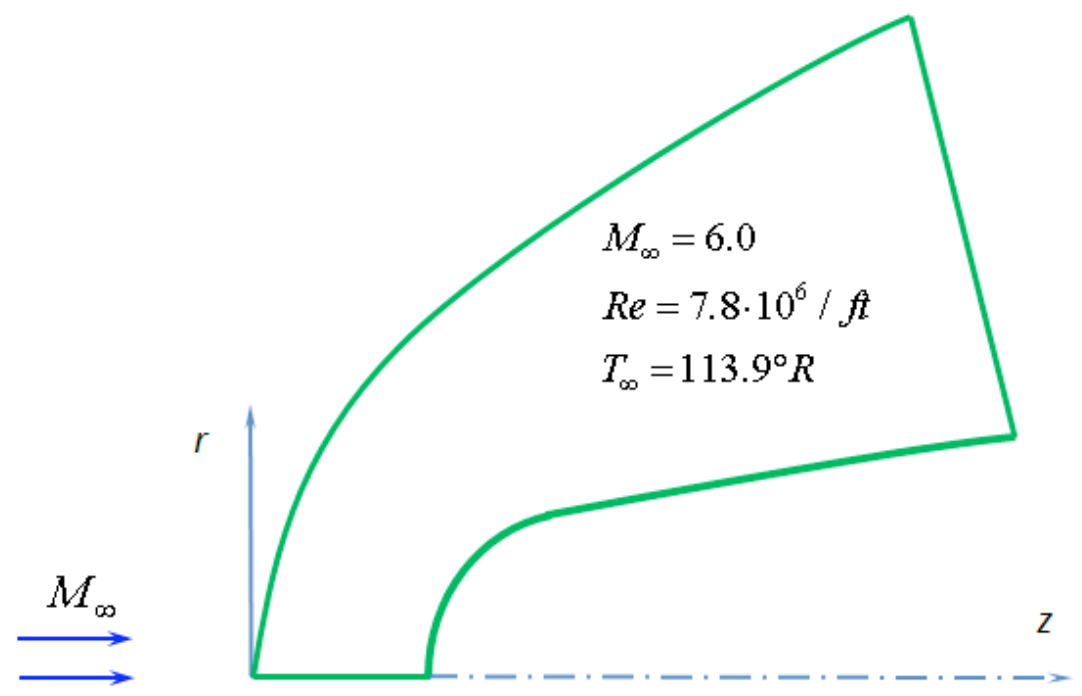

Figure 1. Sketch of the computational domain.

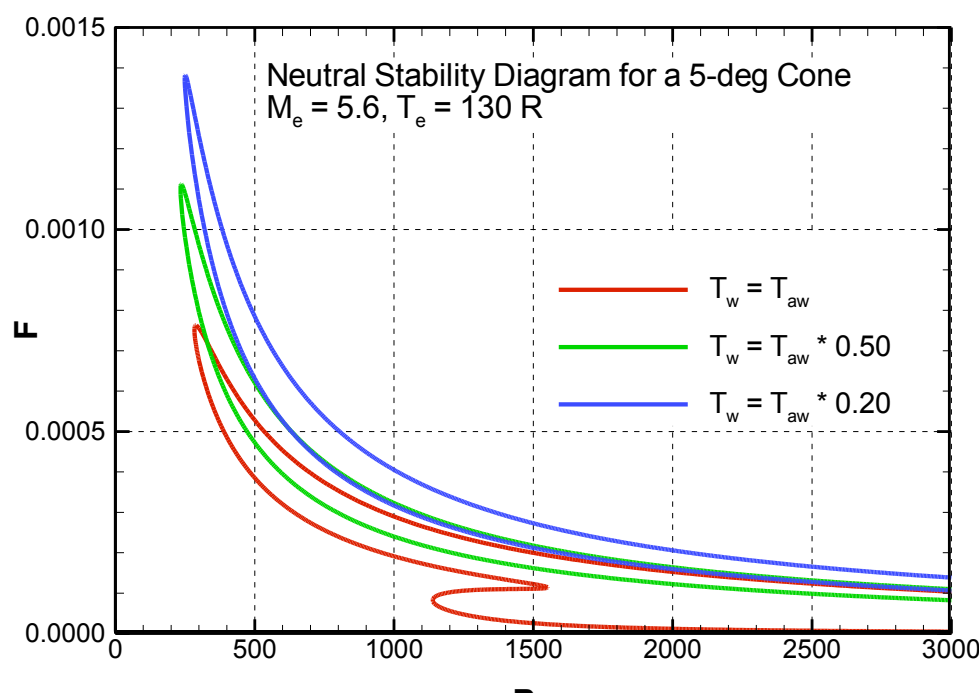

$\mathrm{Re}$

(a)
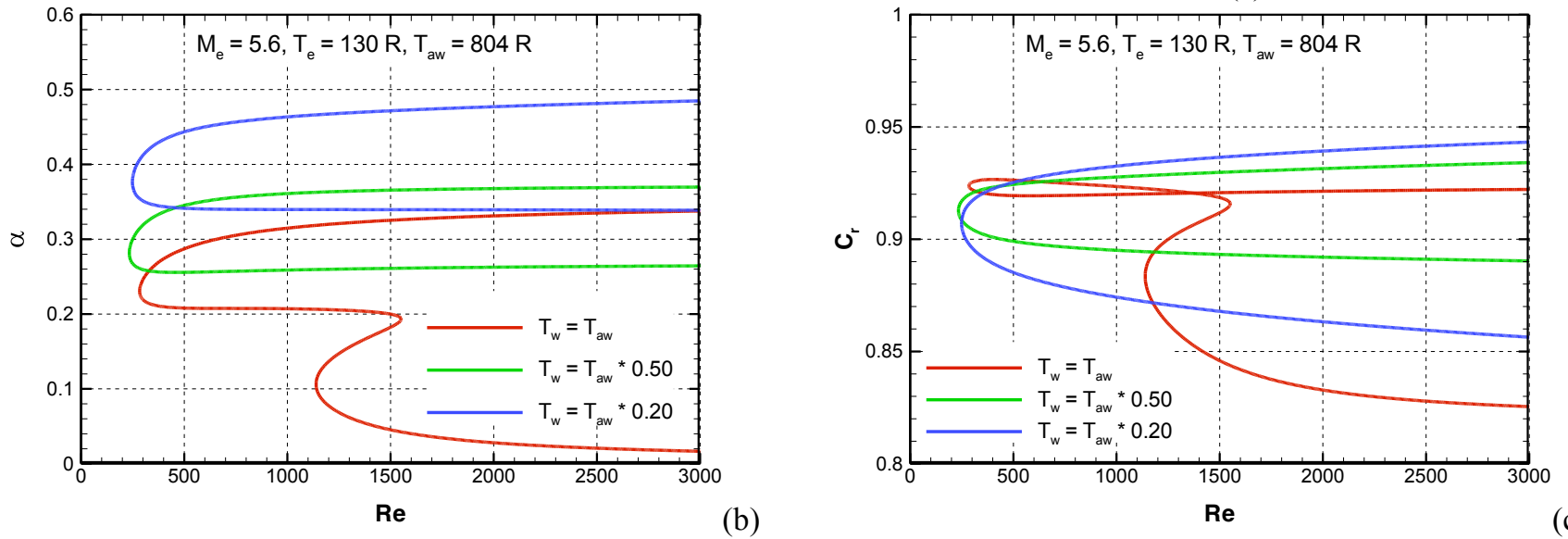

(b)

(c)

Figure 2. Neutral stability diagrams for a 5-deg cone at different wall temperature conditions in (a) $R e-F$, (b) $\operatorname{Re}-\boldsymbol{\alpha}$, and (c) $\mathrm{Re}-\mathrm{C}_{\mathrm{r}}$ planes. 

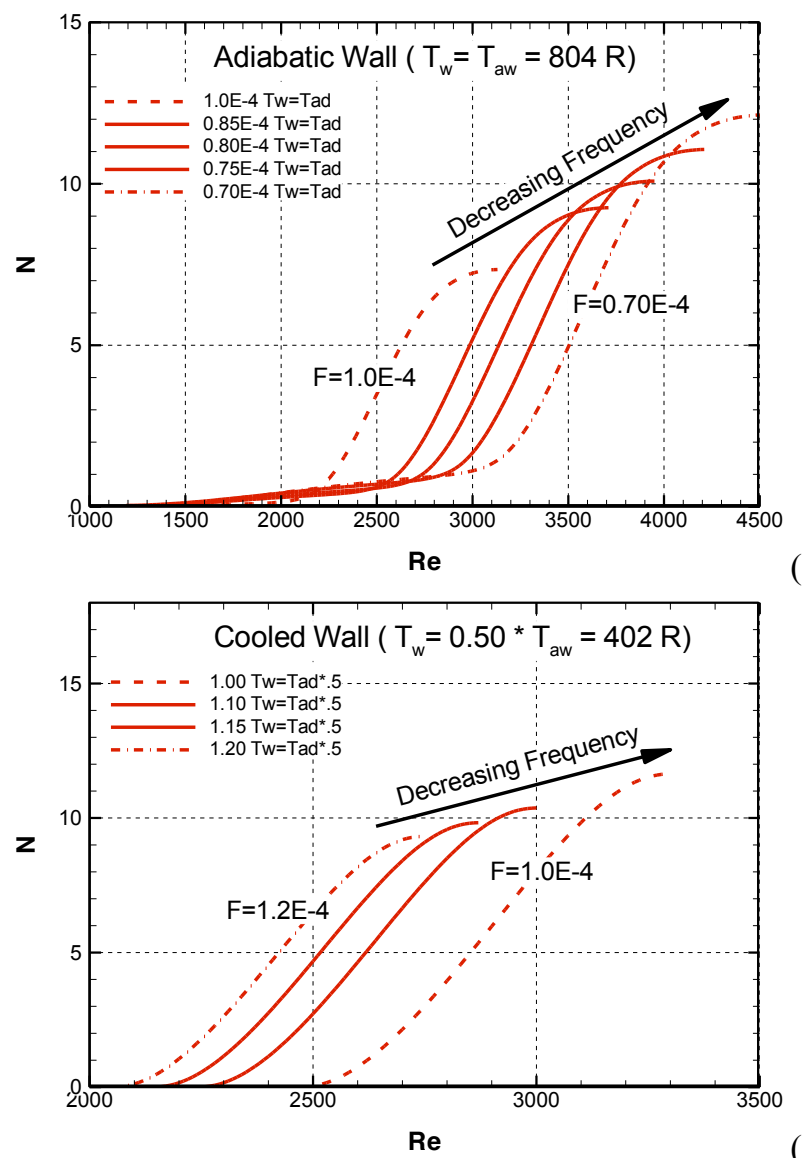

(a)
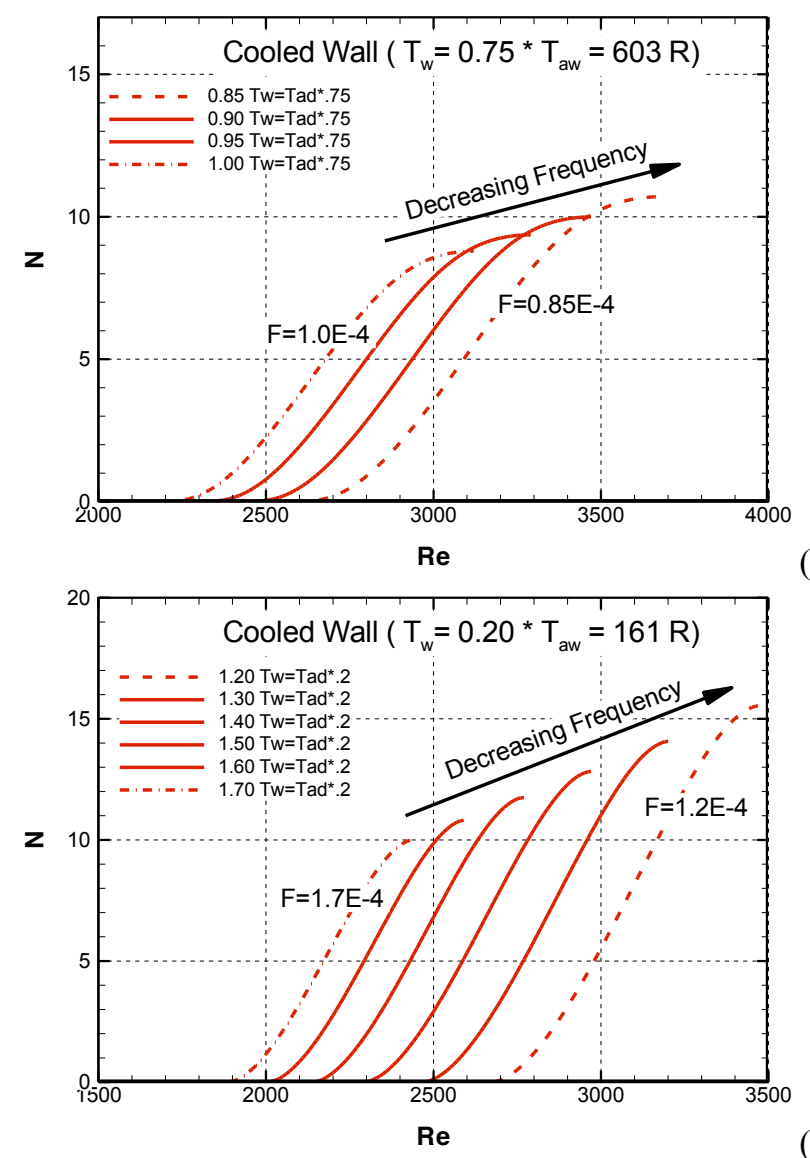

(b)

(d)

Figure 3. N-Factor curves for decreasing frequencies for a blunt cone $\left(r_{n}=0.001\right.$ in.) at different wall temperature conditions. (a) Adiabatic wall ( $T_{\mathrm{aw}}$ ), (b) $\mathrm{T}_{\mathrm{w}}=\mathrm{T}_{\mathrm{aw}}{ }^{\star} 0.75$, (c) $\mathrm{T}_{\mathrm{w}}=\mathrm{T}_{\mathrm{aw}}{ }^{\star} 0.50$, and (d) $\mathrm{T}_{\mathrm{w}}=\mathrm{T}_{\mathrm{aw}}{ }^{\star} 0.20$ 


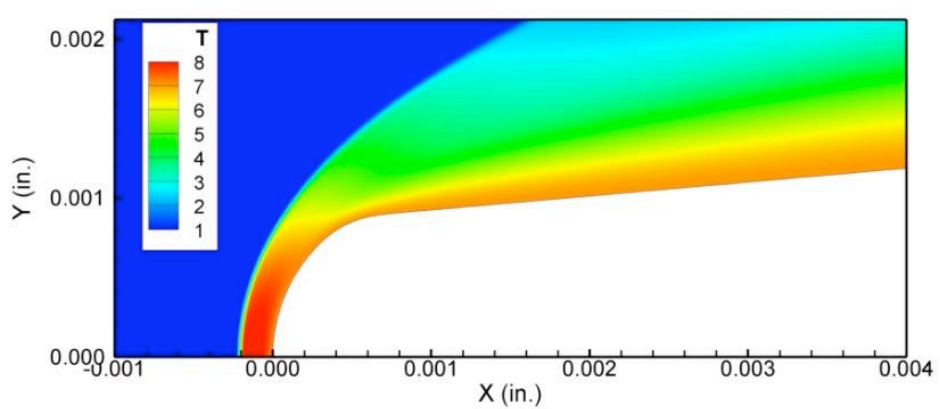

(a) Adiabatic wall $\left(\mathrm{T}_{\mathrm{aw}}\right)$

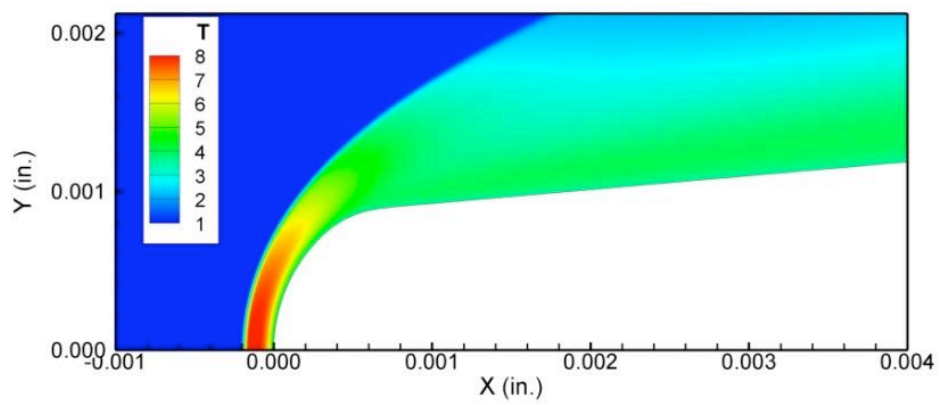

(c) $T_{\mathrm{w}}=\mathrm{T}_{\mathrm{aw}}{ }^{\star} 0.50$

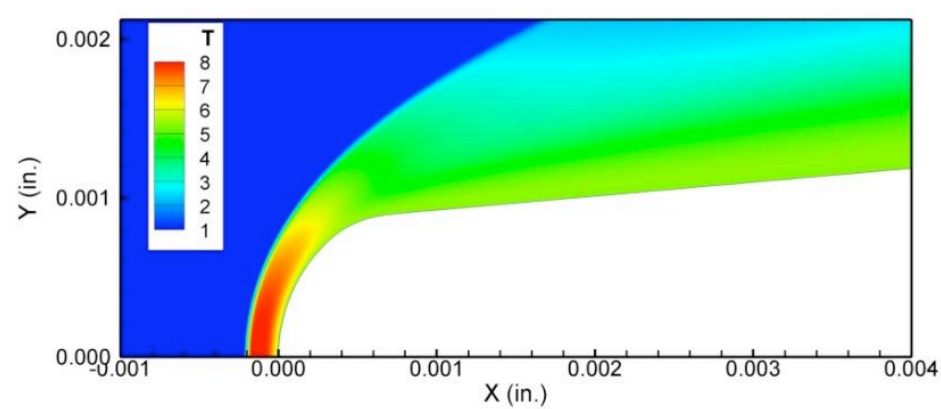

(b) $T_{w}=T_{\text {aw }}{ }^{\star} 0.75$

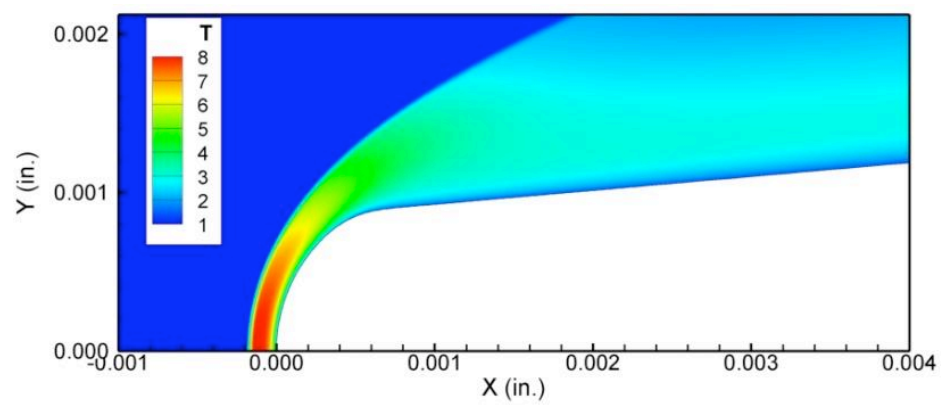

(d) $\mathrm{T}_{\mathrm{w}}=\mathrm{T}_{\mathrm{aw}}{ }^{*} 0.20$

Figure 4. Contours of temperature for flow over a cone with different surface temperatures at $M=6.0$ and $R e=7.8 \times 10^{6}$. (a) Adiabatic wall ( $T_{\text {aw }}$ ), (b) $T_{w}=T_{a w}{ }^{*} 0.75$, (c) $T_{w}=T_{a w}{ }^{\star} 0.50$, and (d) $T_{w}=T_{a w}{ }^{*} 0.20$

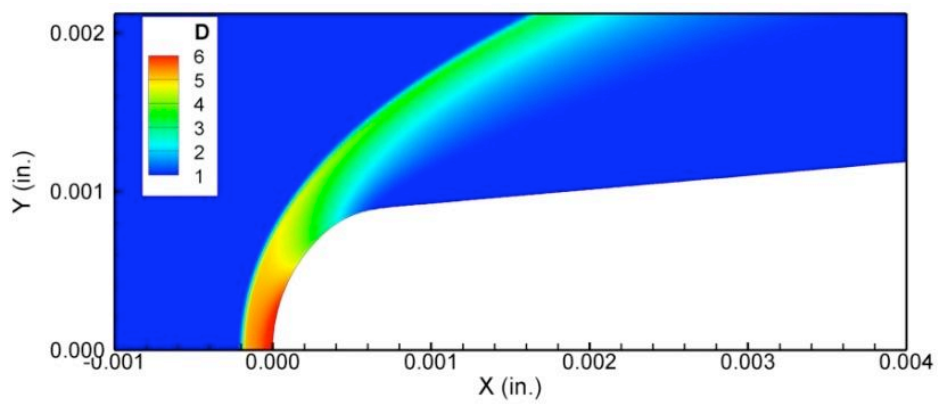

(a) Adiabatic wall $\left(\mathrm{T}_{\mathrm{aw}}\right)$

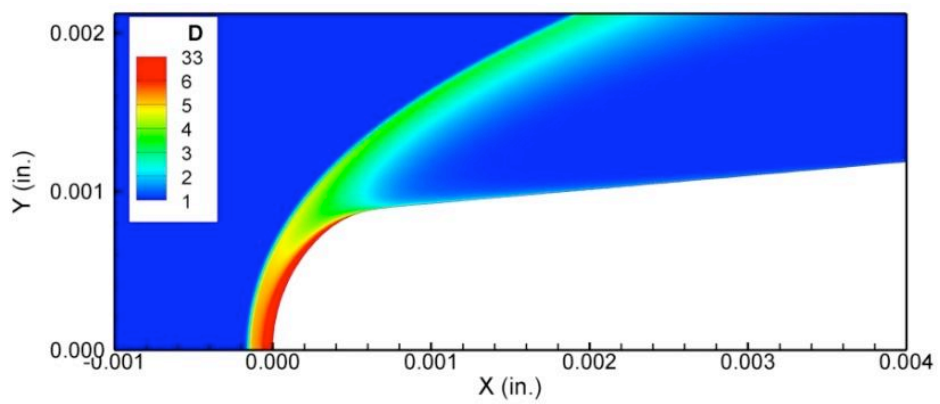

(b) $T_{w}=T_{a w}{ }^{*} 0.20$

Figure 5. Density contours for flow over a cone with different surface temperatures at $M=6.0$ and $R e=7.8 \times 10^{6}$. (a) Adiabatic wall ( $\left.T_{\text {aw }}\right)$, (b) $T_{w}=T_{\text {aw }}{ }^{*} 0.20$

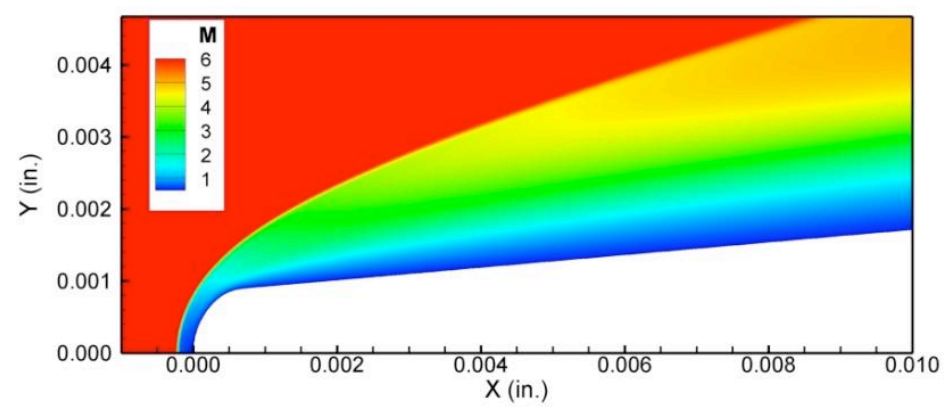

(a) Adiabatic wall $\left(\mathrm{T}_{\mathrm{aw}}\right)$

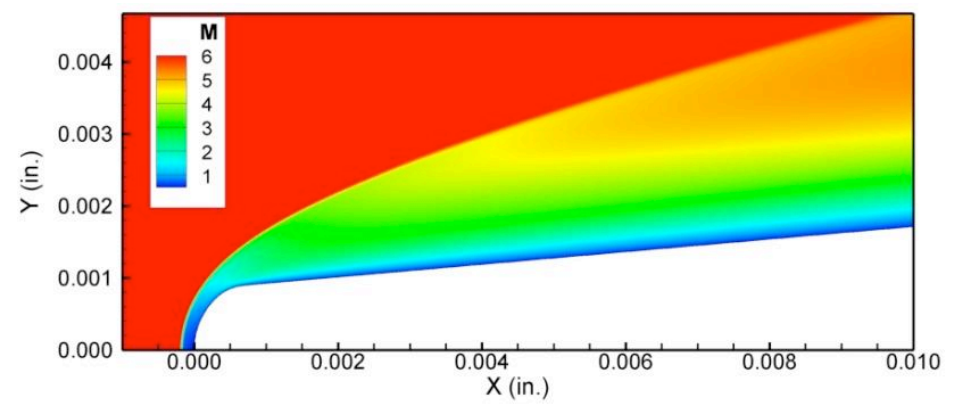

(b) $\mathrm{T}_{\mathrm{w}}=\mathrm{T}_{\mathrm{aw}}{ }^{\star} 0.20$

Figure 6. Mach contours for flow over a cone with different surface temperatures at $M=6.0$ and $R e=7.8 \times 10^{6}$. (a) Adiabatic wall $\left(T_{\mathrm{aw}}\right),(\mathrm{b}) \mathrm{T}_{\mathrm{w}}=\mathrm{T}_{\mathrm{aw}}{ }^{*} 0.20$ 


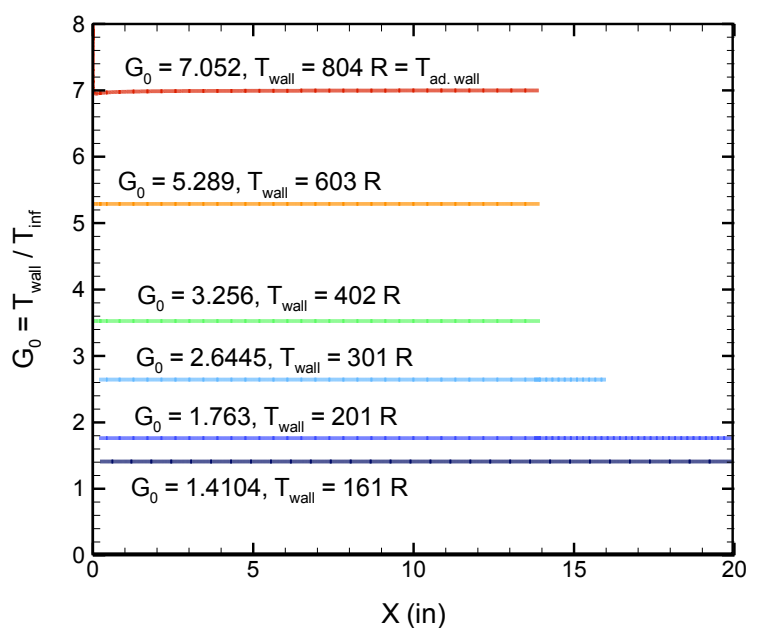

(a)

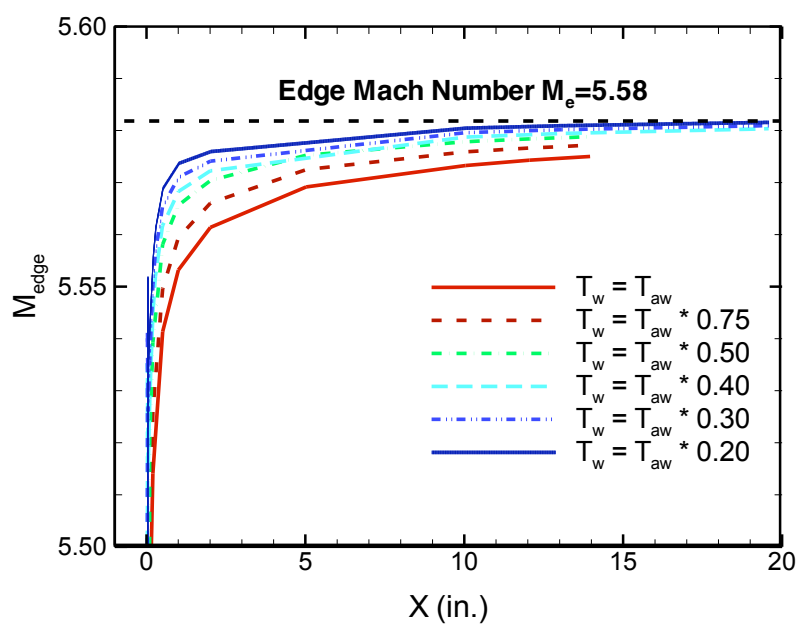

(b)

Figure 7. (a) Wall to free stream temperature ratio along the cone surface. (b) Effect of wall cooling on edge Mach number.

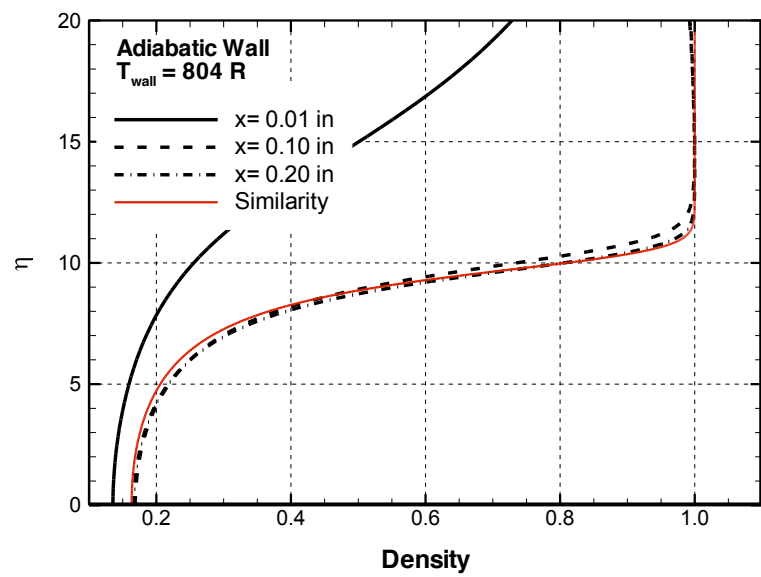

(a) Adiabatic wall ( $\left.T_{\text {aw }}\right)$

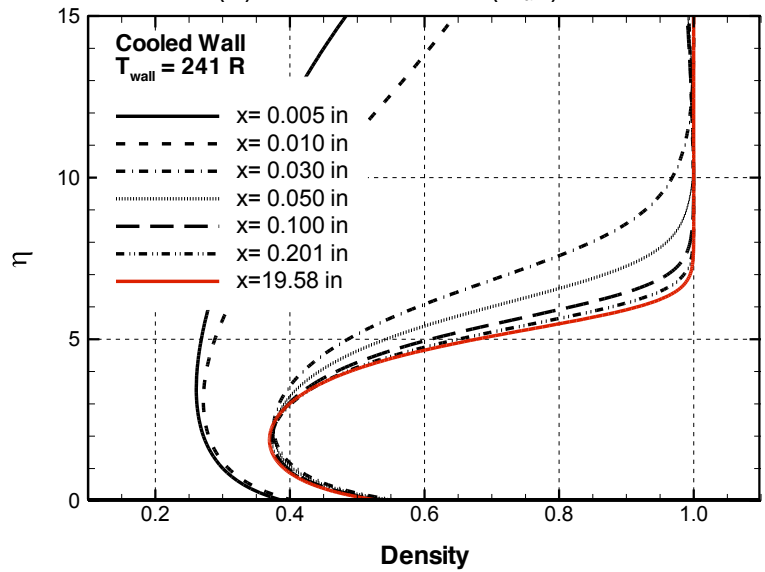

(c) $T_{\mathrm{w}}=\mathrm{T}_{\mathrm{aw}}{ }^{*} 0.30$

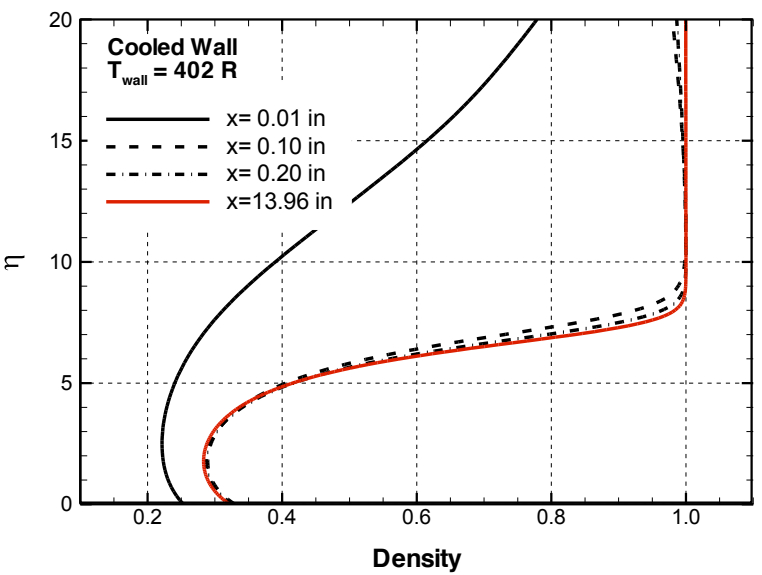

(b) $\mathrm{T}_{\mathrm{w}}=\mathrm{T}_{\mathrm{aw}}{ }^{\star} 0.50$

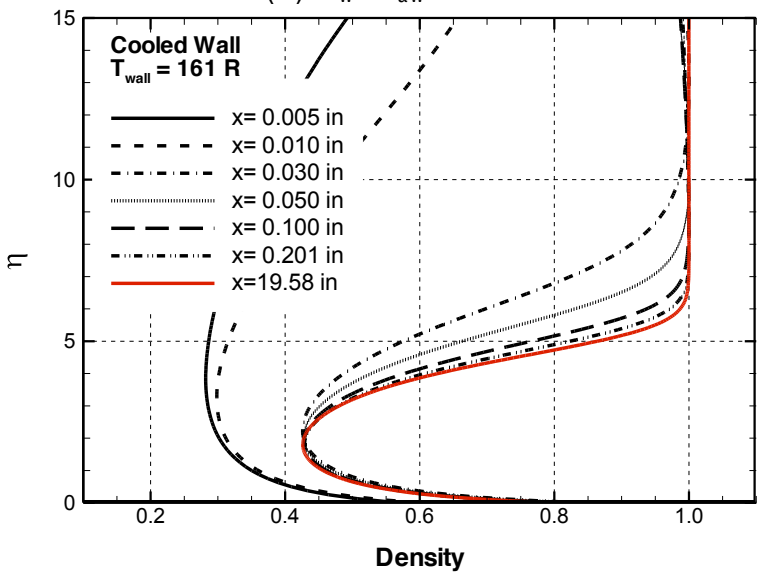

(d) $\mathrm{T}_{\mathrm{w}}=\mathrm{T}_{\mathrm{aw}}{ }^{\star} 0.20$

Figure 8. Mean flow density profiles at different $X$ locations in similarity coordinates for different wall temperatures. (a) Adiabatic wall ( $\mathrm{T}_{\mathrm{aw}}$ ), (b) $\mathrm{T}_{\mathrm{w}}=\mathrm{T}_{\mathrm{aw}}{ }^{\star} 0.50$, (c) $\mathrm{T}_{\mathrm{w}}=\mathrm{T}_{\mathrm{aw}}{ }^{\star} 0.30$, and (d) $\mathrm{T}_{\mathrm{w}}=\mathrm{T}_{\mathrm{aw}}{ }^{\star} 0.20$. 


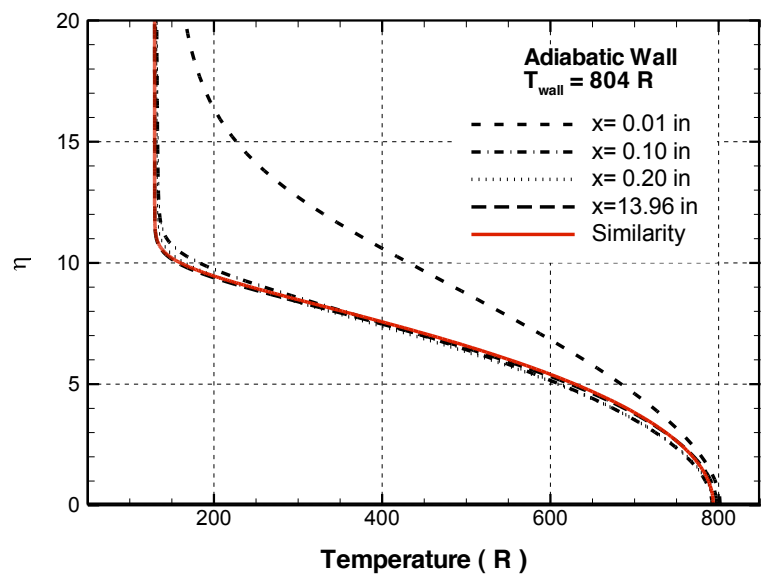

(a) Adiabatic wall ( $\left.T_{\text {aw }}\right)$

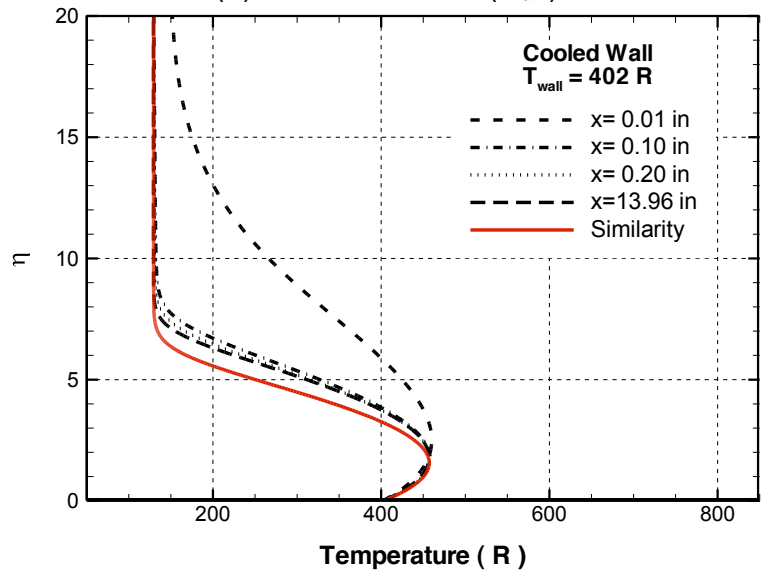

(c) $T_{\mathrm{w}}=\mathrm{T}_{\mathrm{aw}}{ }^{\star} 0.50$

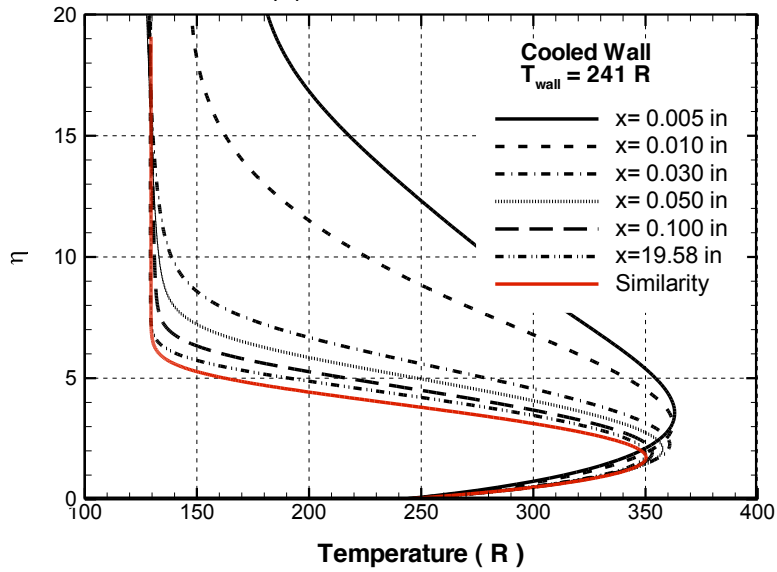

(e) $\mathrm{T}_{\mathrm{w}}=\mathrm{T}_{\mathrm{aw}}{ }^{*} 0.30$

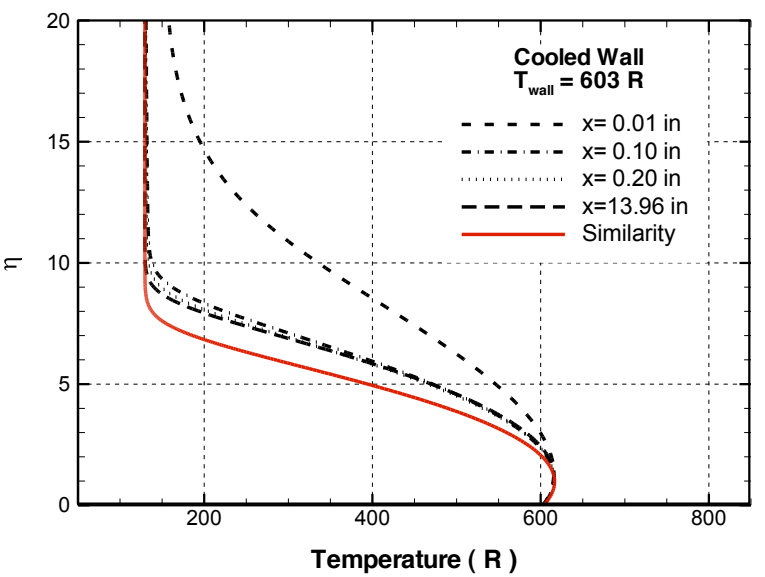

(b) $\mathrm{T}_{\mathrm{w}}=\mathrm{T}_{\mathrm{aw}}{ }^{*} 0.75$

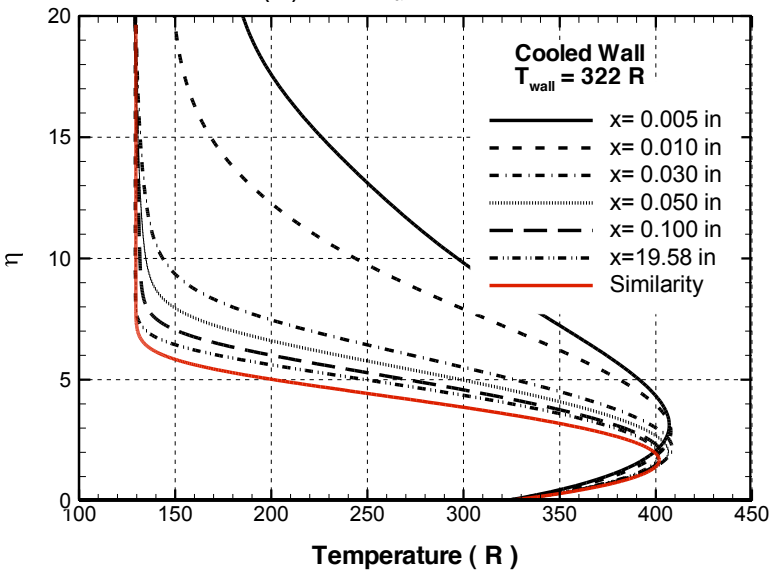

(d) $\mathrm{T}_{\mathrm{w}}=\mathrm{T}_{\mathrm{aw}}{ }^{*} 0.40$

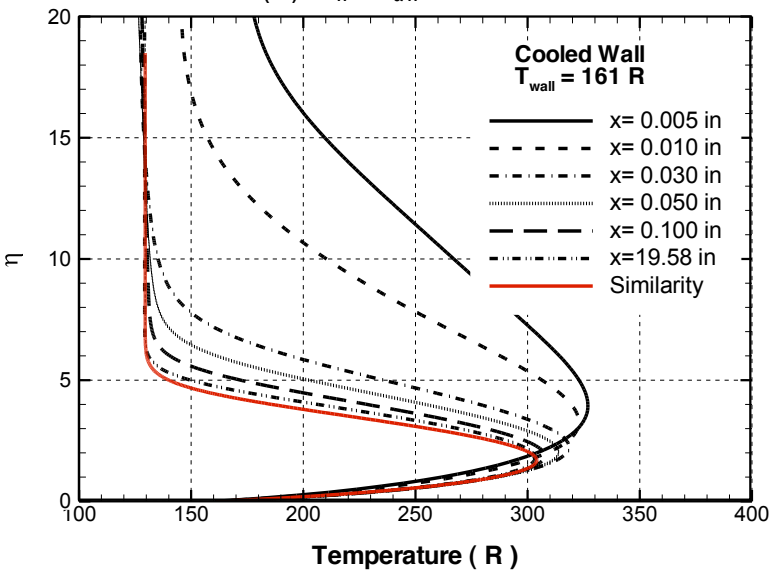

(f) $T_{w}=T_{a w}{ }^{*} 0.20$

Figure 9. Mean flow temperature profiles at different $X$ locations in similarity coordinates for different wall temperatures. (a) Adiabatic wall ( $T_{\mathrm{aw}}$ ), (b) $\mathrm{T}_{\mathrm{w}}=\mathrm{T}_{\mathrm{aw}}{ }^{\star} 0.75$, (c) $\mathrm{T}_{\mathrm{w}}=\mathrm{T}_{\mathrm{aw}}{ }^{\star} 0.50$, (d) $\mathrm{T}_{\mathrm{w}}=\mathrm{T}_{\mathrm{aw}}{ }^{\star} 0.40$, (e) $\mathrm{T}_{\mathrm{w}}=\mathrm{T}_{\mathrm{aw}}{ }^{\star} 0.30$, and (f) $T_{w}=T_{\text {aw }}{ }^{*} 0.20$. 


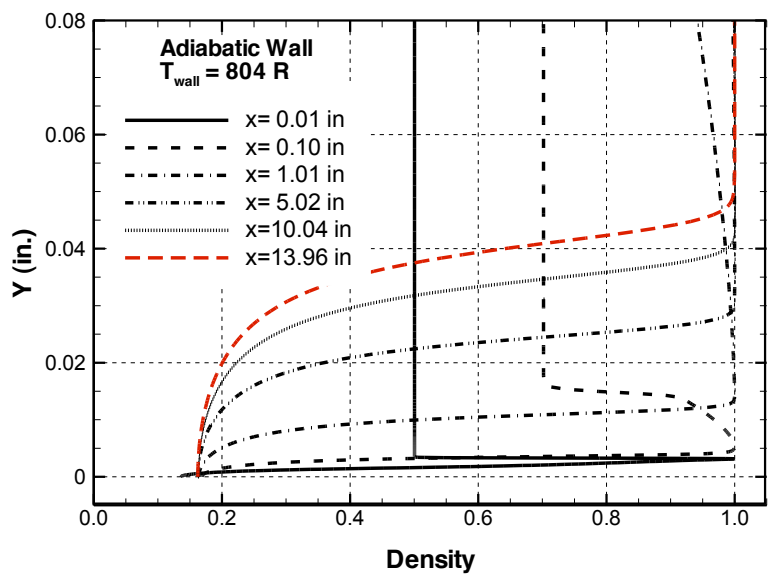

(a) Adiabatic wall ( $\left.\mathrm{T}_{\mathrm{aw}}\right)$

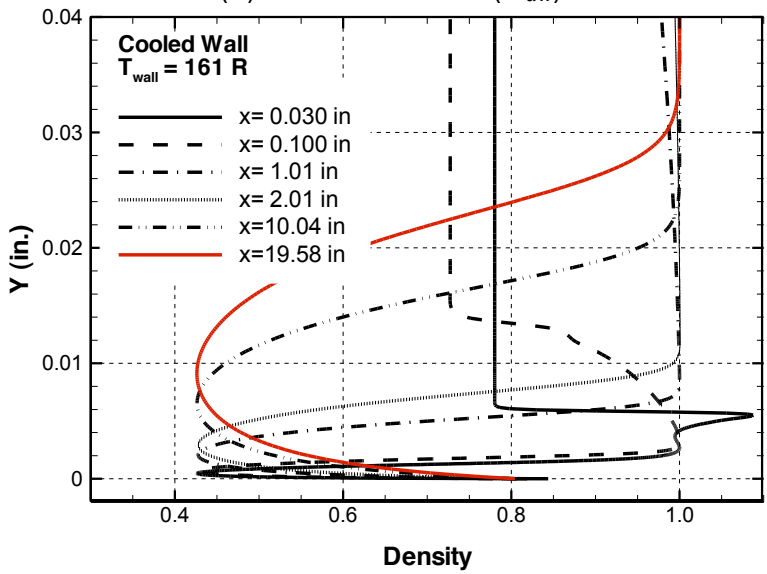

(b) $\mathrm{T}_{\mathrm{w}}=\mathrm{T}_{\mathrm{aw}}{ }^{\star} 0.20$

Figure 10. Mean flow density profiles at different $X$ locations in physical coordinates for (a) Adiabatic wall $\left(T_{a w}\right)$, and $(b) T_{w}=T_{a w}{ }^{\star} 0.20$.

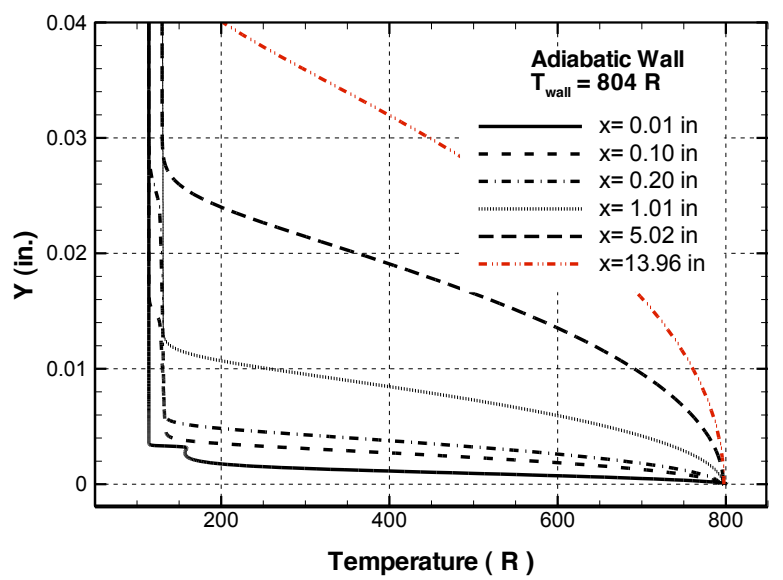

(a) Adiabatic wall ( $\left.T_{\text {aw }}\right)$

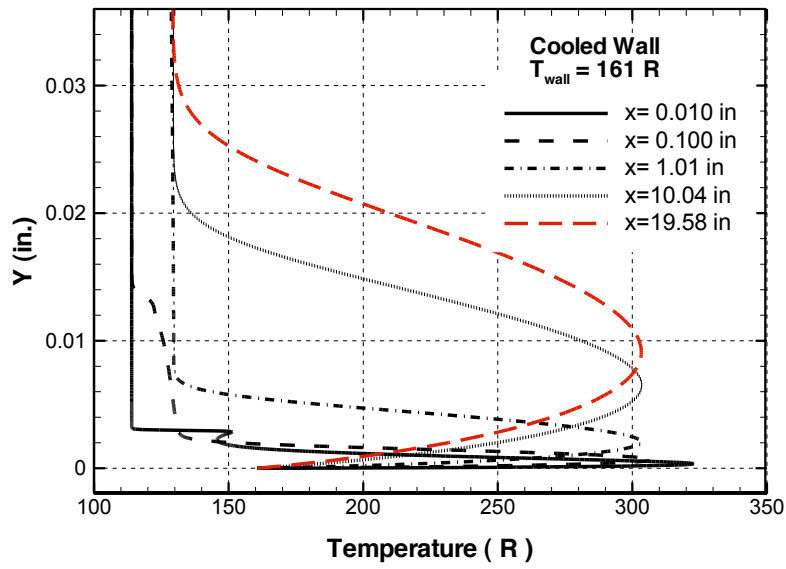

(b) $\mathrm{T}_{\mathrm{w}}=\mathrm{T}_{\mathrm{aw}}{ }^{\star} 0.20$

Figure 11. Mean flow temperature profiles at different $X$ locations in physical coordinates for (a) Adiabatic wall $\left(T_{\mathrm{aw}}\right)$, and (b) $\mathrm{T}_{\mathrm{w}}=\mathrm{T}_{\mathrm{aw}}{ }^{\star} 0.20$. 


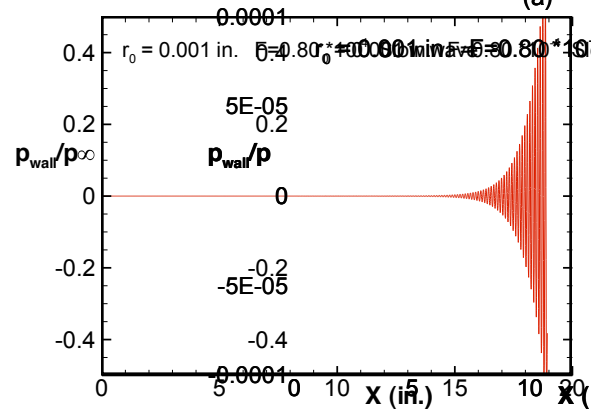

(a) Adiabatic wall $\left(\mathrm{T}_{\mathrm{aw}}\right)$

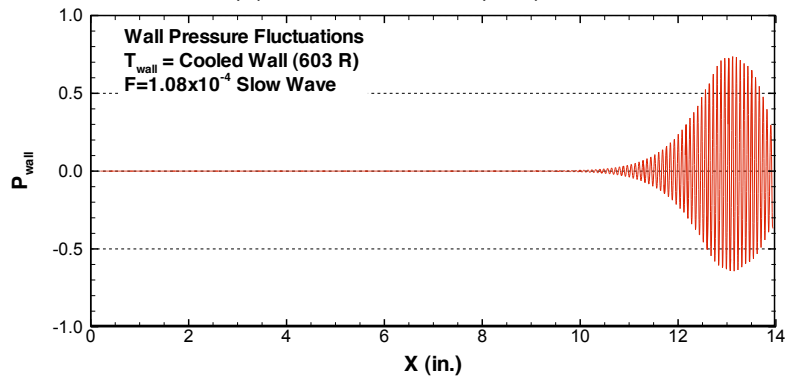

(b) $T_{w}=T_{a w}{ }^{*} 0.75$

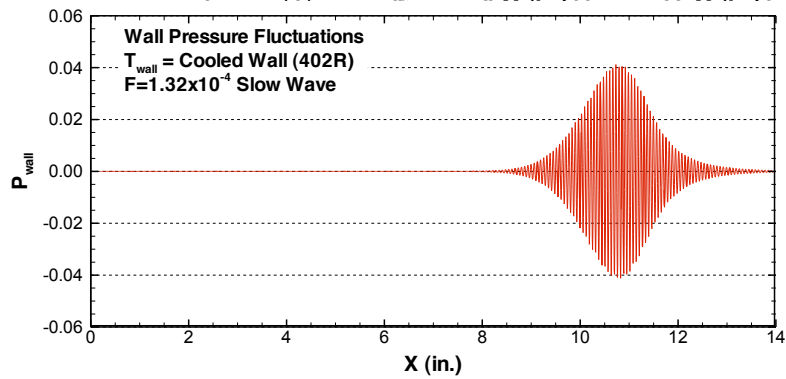

(c) $T_{\mathrm{w}}=\mathrm{T}_{\mathrm{aw}}{ }^{*} 0.50$

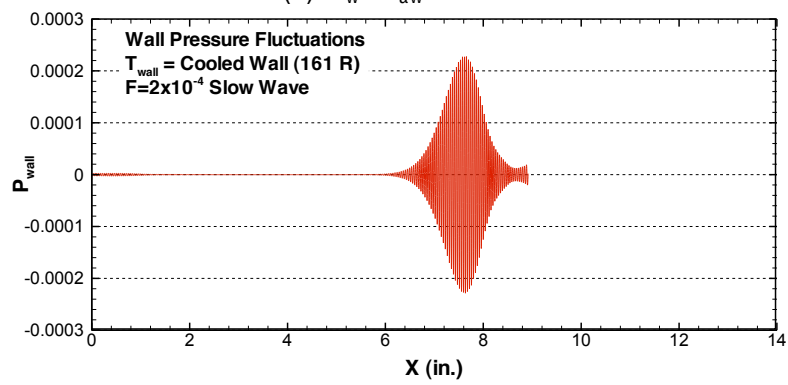

(d) $T_{w}=T_{a w}{ }^{\star} 0.20$

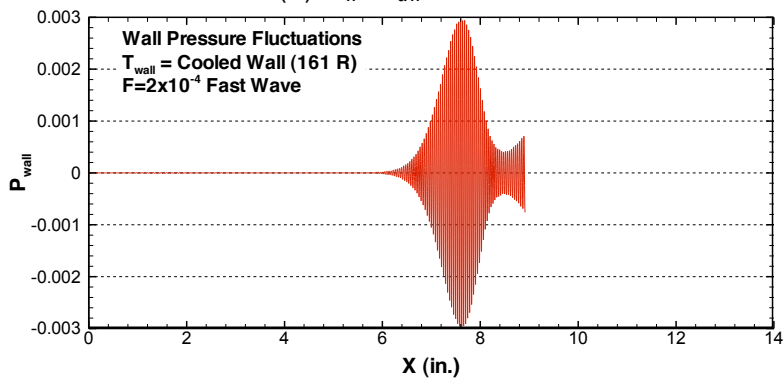

(e) $T_{w}=T_{a w}{ }^{\star} 0.20$

Figure 12. Pressure fluctuations on the wall, (a) Adiabatic wall ( $T_{a w}$ ), (b) $T_{w}=T_{a w}{ }^{*} 0.75$, (c) $T_{w}=T_{a w}{ }^{*} 0.50, \quad$ (d) $\mathrm{T}_{\mathrm{w}}=\mathrm{T}_{\mathrm{aw}}{ }^{\star} 0.20$, and (e) $\mathrm{T}_{\mathrm{w}}=\mathrm{T}_{\mathrm{aw}}{ }^{\star} 0.20$ (Fast Wave).

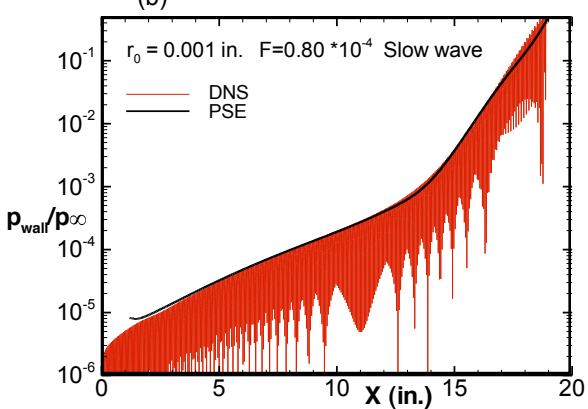

(a) Adiabatic wall $\left(\mathrm{T}_{\mathrm{aw}}\right)$

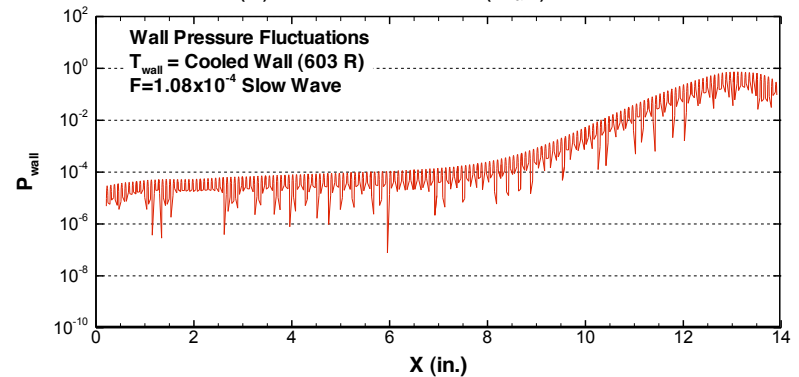

(b) $\mathrm{T}_{\mathrm{w}}=\mathrm{T}_{\mathrm{aw}}{ }^{\star} 0.75$

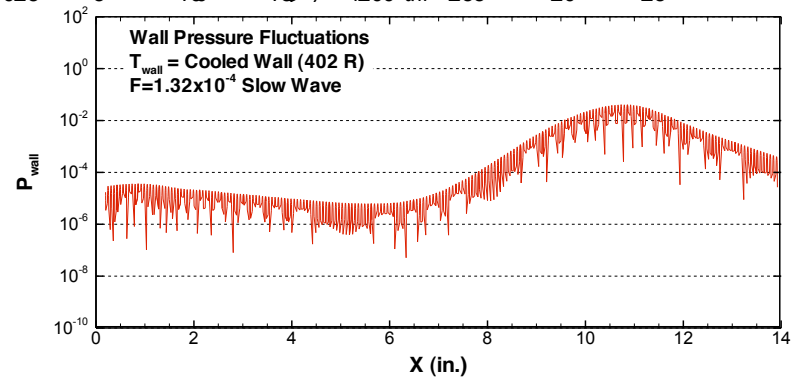

(c) $T_{\mathrm{w}}=\mathrm{T}_{\mathrm{aw}}{ }^{\star} 0.50$

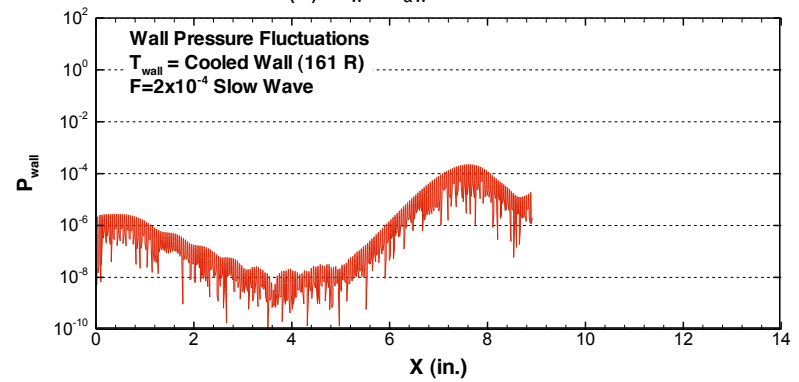

(d) $\mathrm{T}_{\mathrm{w}}=\mathrm{T}_{\mathrm{aw}}{ }^{\star} 0.20$

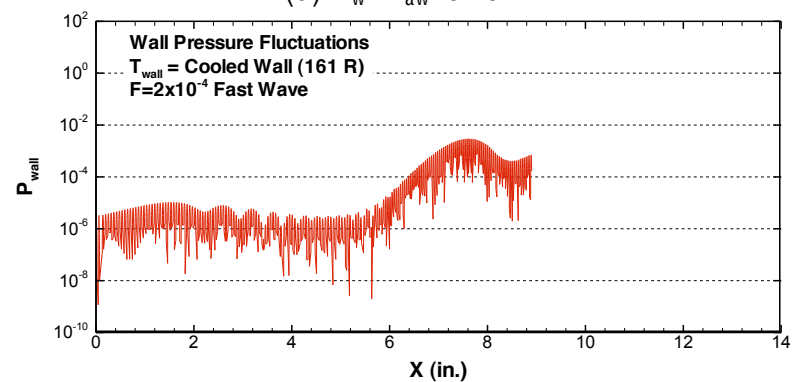

(e) $T_{w}=T_{a w}{ }^{*} 0.20$

Figure 13. Pressure fluctuations on the wall in Log Scale,

(a) Adiabatic wall ( $T_{\mathrm{aw}}$ ), (b) $\mathrm{T}_{\mathrm{w}}=\mathrm{T}_{\mathrm{aw}}{ }^{*} 0.75$, (c) $\mathrm{T}_{\mathrm{w}}=\mathrm{T}_{\mathrm{aw}}{ }^{\star} 0.50$,

(d) $\mathrm{T}_{\mathrm{w}}=\mathrm{T}_{\mathrm{aw}}{ }^{\star} 0.20$, and (e) $\mathrm{T}_{\mathrm{w}}=\mathrm{T}_{\mathrm{aw}}{ }^{\star} 0.20$ (Fast Wave). 


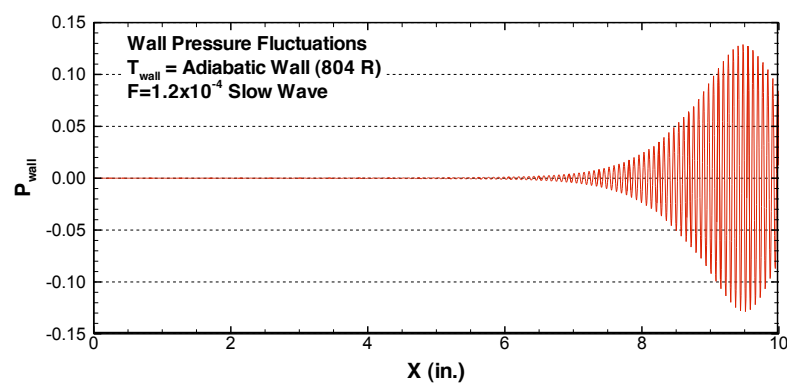

(a) Adiabatic wall $\left(T_{\text {aw }}\right)$

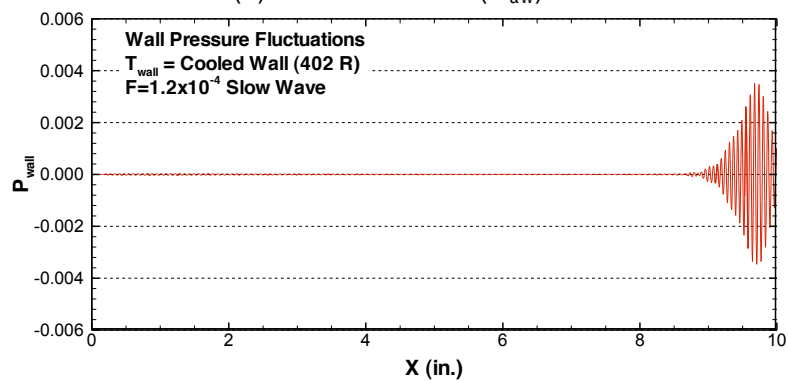

(b) $T_{w}=T_{a w}{ }^{*} 0.50$

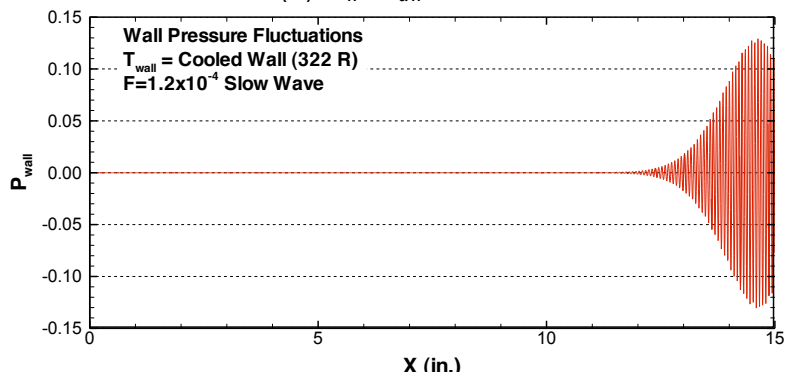

(c) $T_{\mathrm{w}}=\mathrm{T}_{\mathrm{aw}}{ }^{\star} 0.40$

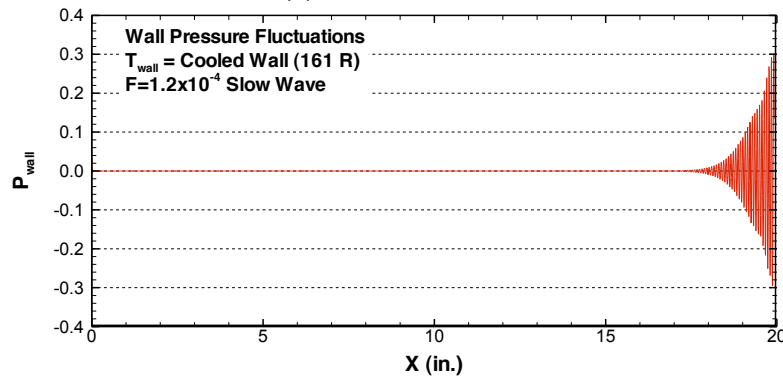

(d) $T_{w}=T_{a w}{ }^{*} 0.20$

Figure 14. Pressure fluctuations on the wall under the effect of same forcing frequency $F=1.2^{*} 10^{-4}$, (a) Adiabatic wall ( $T_{\mathrm{aw}}$ ), (b) $\mathrm{T}_{\mathrm{w}}=\mathrm{T}_{\mathrm{aw}}{ }^{*} 0.50$, (c) $\mathrm{T}_{\mathrm{w}}=\mathrm{T}_{\mathrm{aw}}{ }^{*} 0.40$, and (d) $\mathrm{T}_{\mathrm{w}}=\mathrm{T}_{\mathrm{aw}}{ }^{\star} 0.20$.

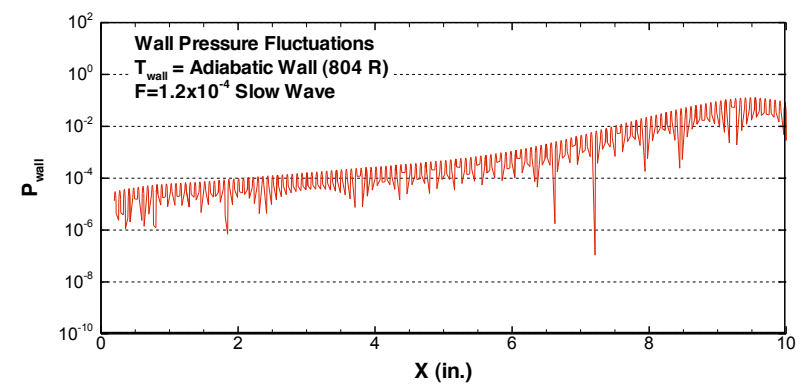

(a) Adiabatic wall ( $\left.T_{\text {aw }}\right)$

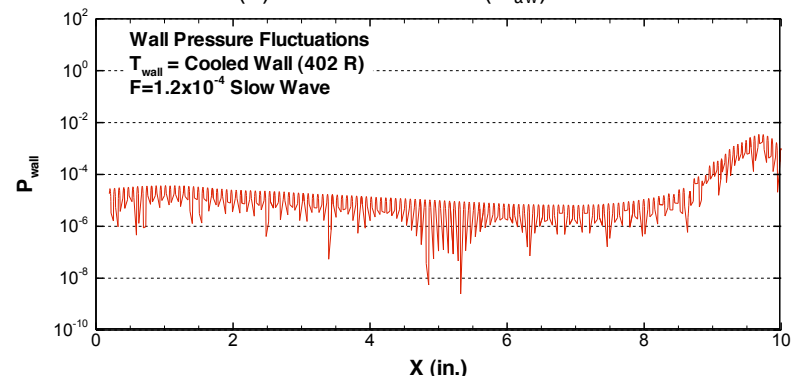

(b) $T_{w}=T_{a w}{ }^{\star} 0.50$

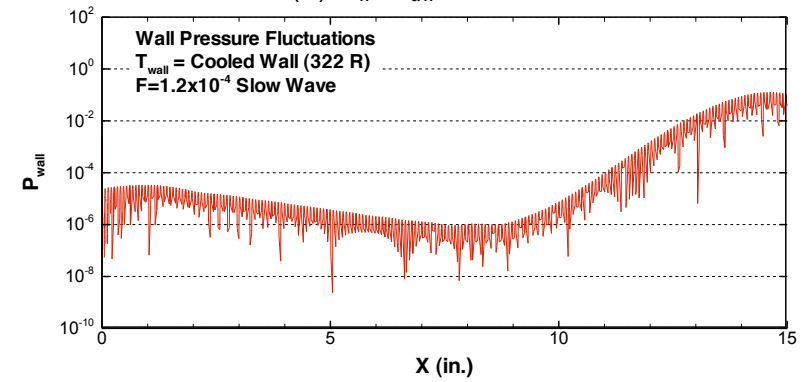

(c) $T_{w}=T_{a w}{ }^{\star} 0.40$

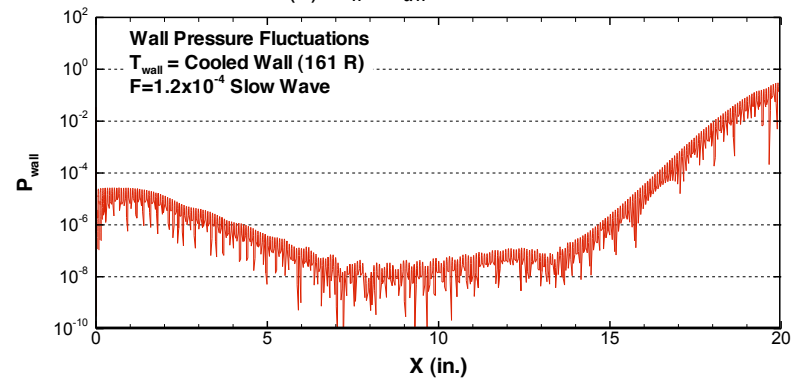

(d) $T_{w}=T_{\text {aw }}{ }^{\star} 0.20$

Figure 15. Pressure fluctuations in log scale on the wall under the effect of same forcing frequency $F=1.2^{*} 10^{-4}$, (a) Adiabatic wall ( $T_{\mathrm{aw}}$ ), (b) $\mathrm{T}_{\mathrm{w}}=\mathrm{T}_{\mathrm{aw}}{ }^{*} 0.50$, (c) $\mathrm{T}_{\mathrm{w}}=\mathrm{T}_{\mathrm{aw}}{ }^{\star} 0.40$, and (d) $\mathrm{T}_{\mathrm{w}}=\mathrm{T}_{\mathrm{aw}}{ }^{\star} 0.20$. 


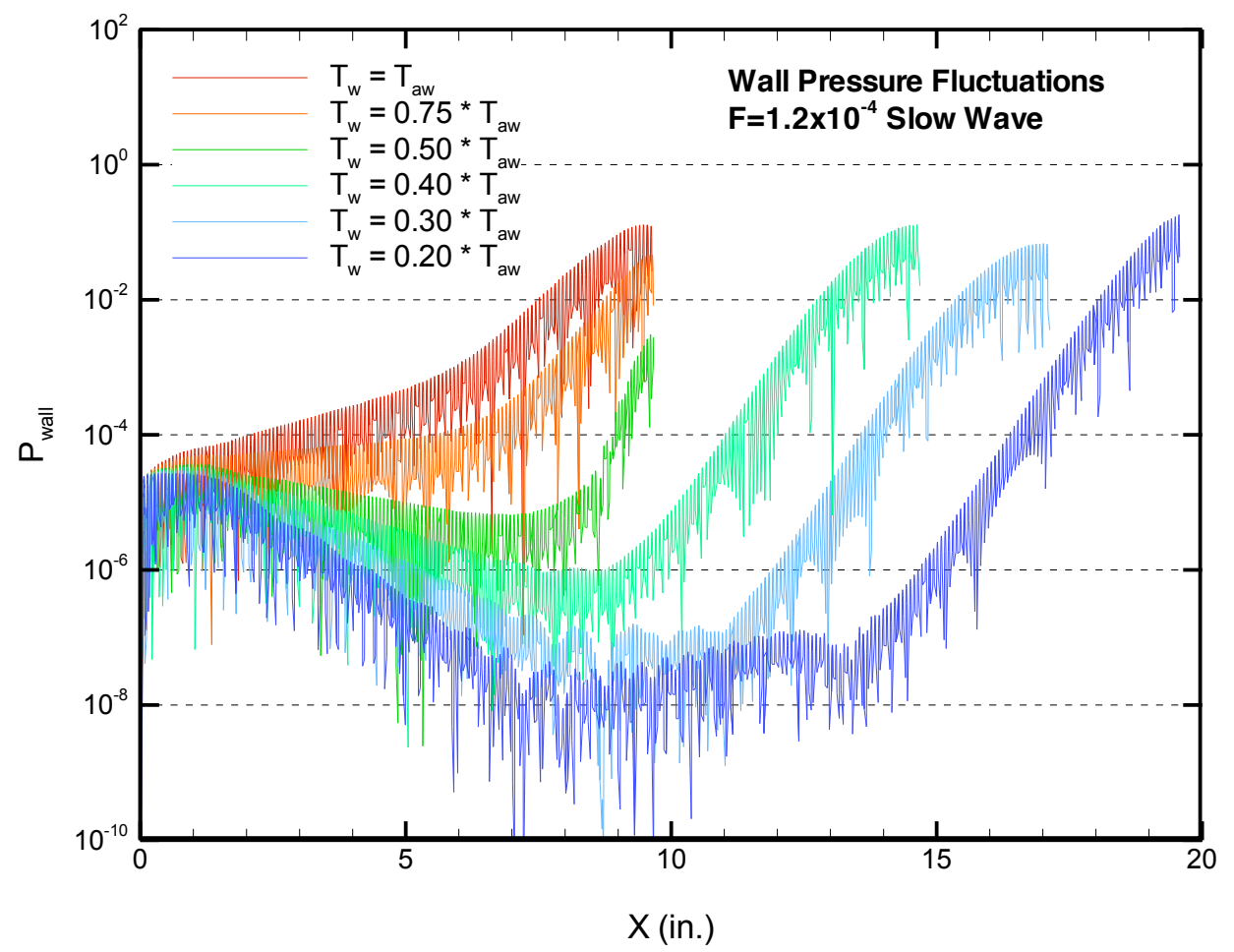

Figure 16. Comparison of wall pressure fluctuations under the same forcing frequency $\left(F=1.2^{*} 10^{-4}\right)$ at different wall temperature conditions.

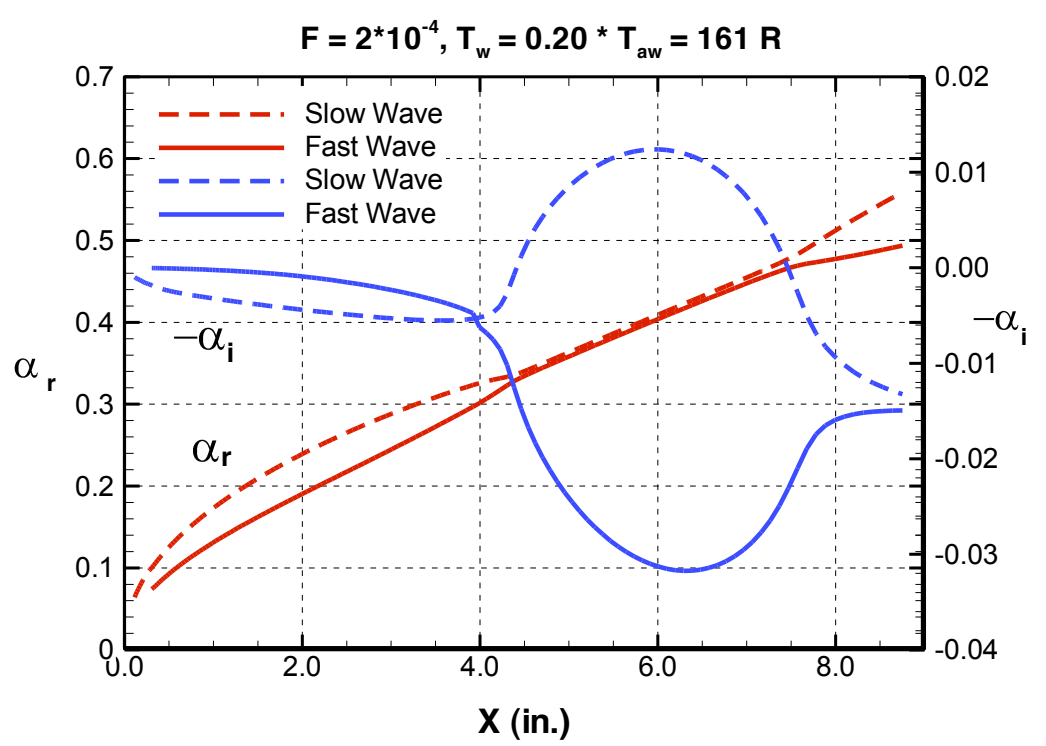

Figure 17. Eigenvalues $\left(\boldsymbol{\alpha}_{\mathrm{r}}, \boldsymbol{\alpha}_{\mathrm{i}}\right)$ for fast and slow modes for $\mathrm{F}=2.0^{\star} 10^{-4}$. 


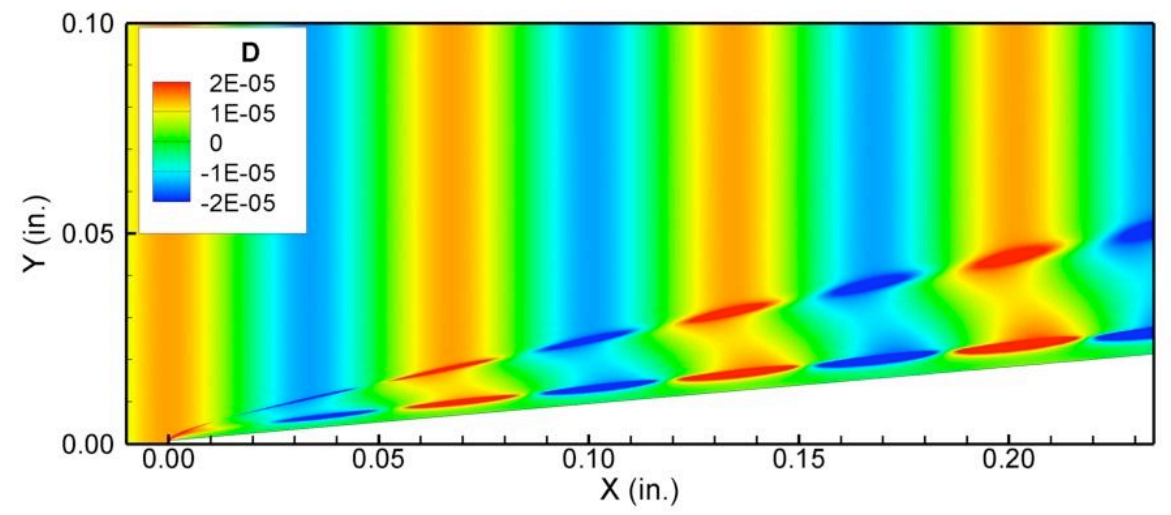

(a) Adiabatic wall $\left(\mathrm{T}_{\mathrm{aw}}\right)$

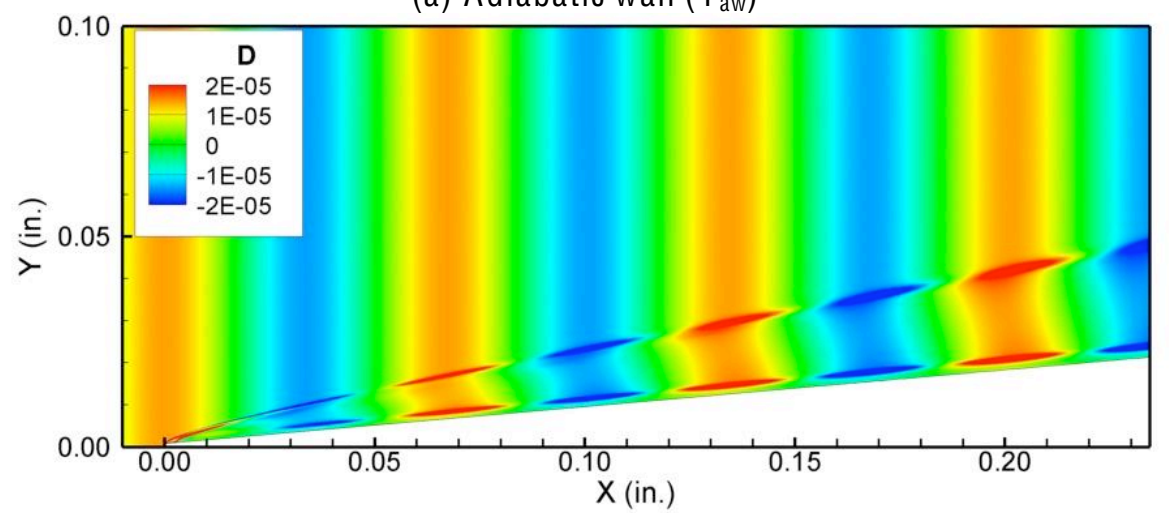

(b) Cooled wall $\left(T_{\mathrm{w}}=\mathrm{T}_{\mathrm{aw}}{ }^{\star} 0.20\right)$

Figure 18. Contours of unsteady density fluctuations inside the boundary layer near the nose region.

(a) Adiabatic wall $\left(T_{w}=T_{a w}\right)$, (b) Cooled wall $\left(T_{w}=T_{a w}{ }^{\star} 0.20\right)$ 


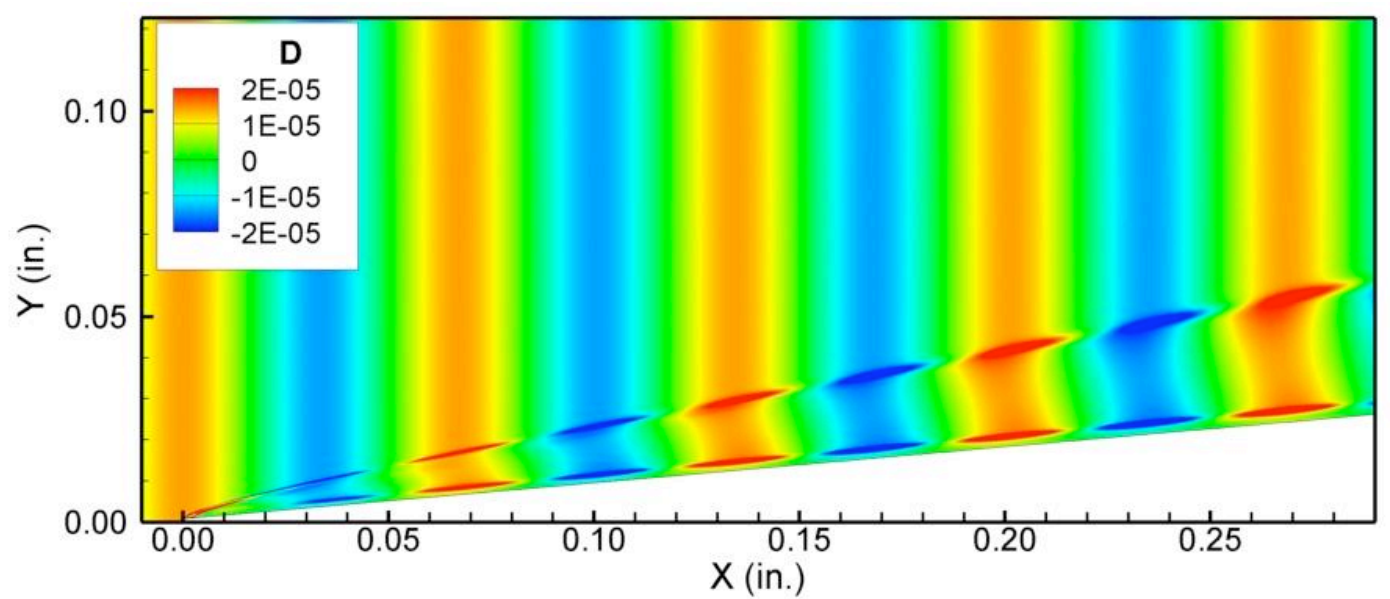

(a) $x=[0.0-0.3]$ in

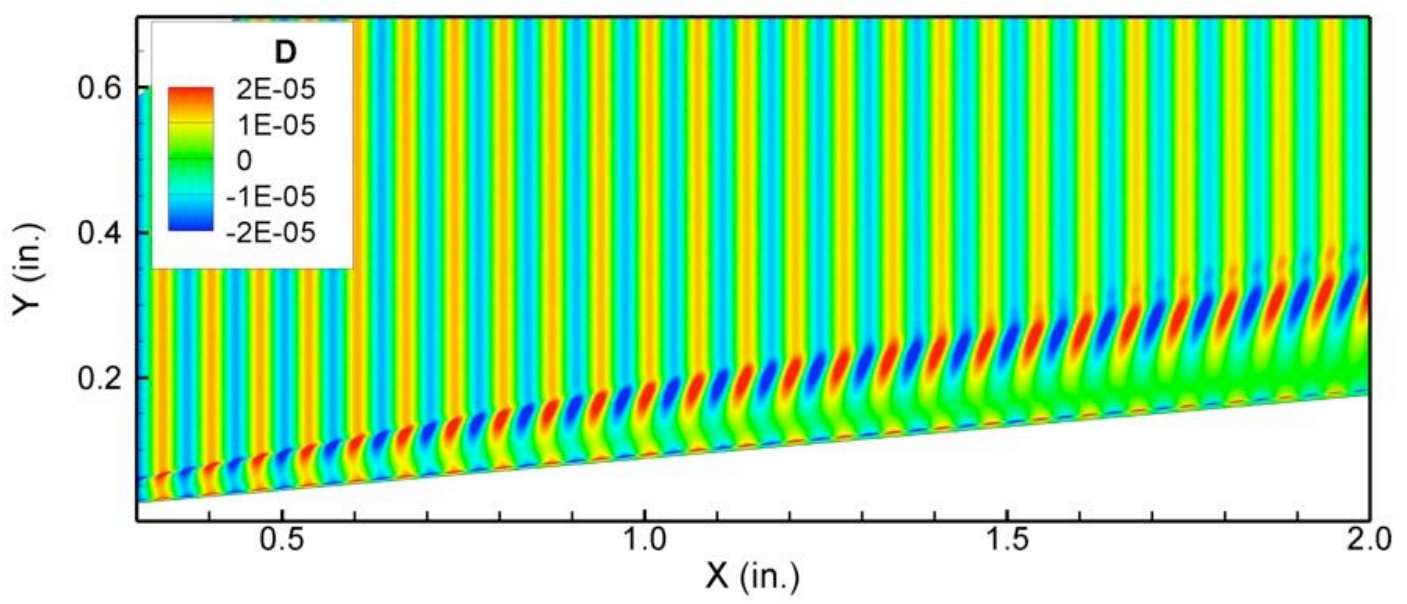

(b) $x=[0.3-2.0]$ in

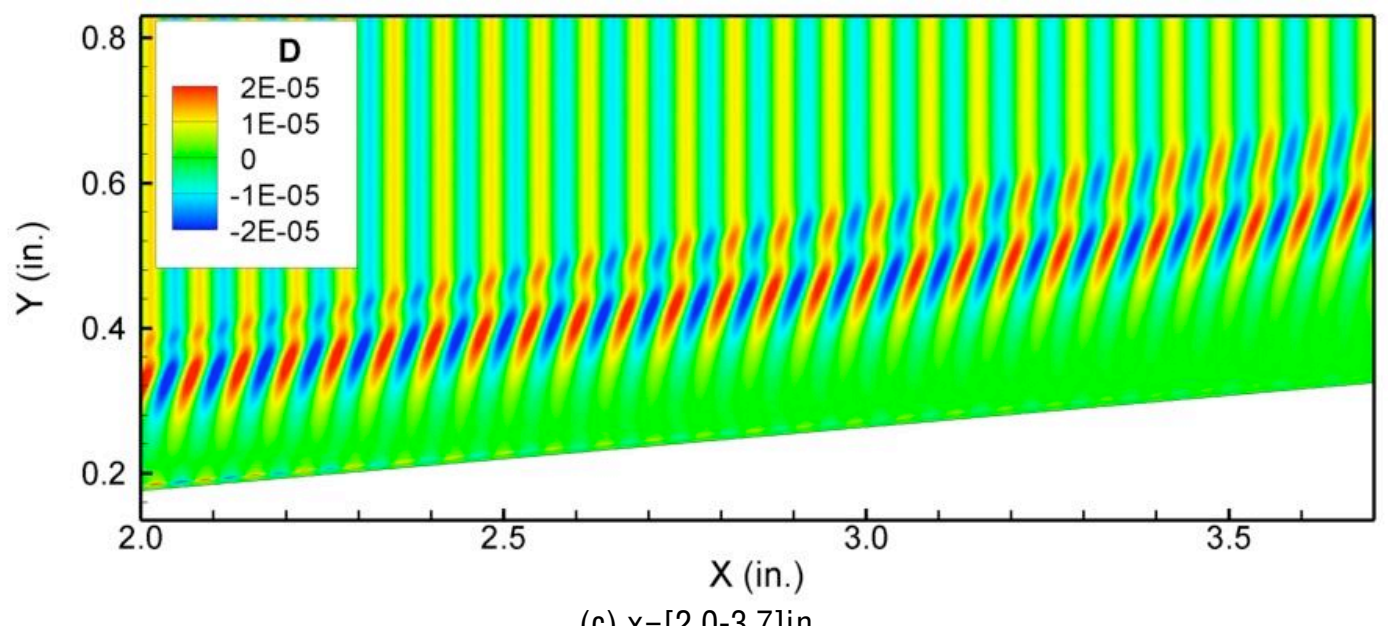

Figure 19. Contours of unsteady density fluctuations inside the boundary layer along the cooled cone wall $\left(\mathrm{T}_{\mathrm{w}}=\mathrm{T}_{\mathrm{aw}}{ }^{\star} 0.20\right)$, (a) $\mathrm{x}=[0.0-0.3] \mathrm{in},(\mathrm{a}) \mathrm{x}=[0.3-2.0] \mathrm{in}$, and (a) $\mathrm{x}=[2.0-3.7] \mathrm{in}$. 


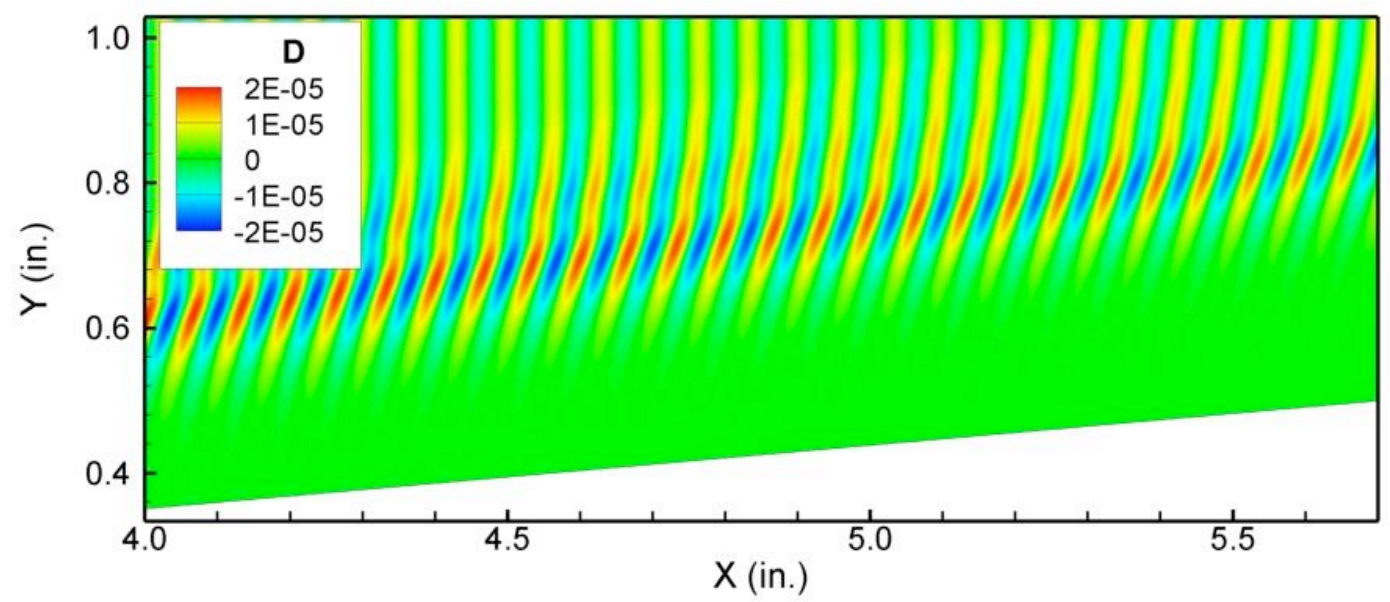

(a) $x=[4.0-5.7]$ in

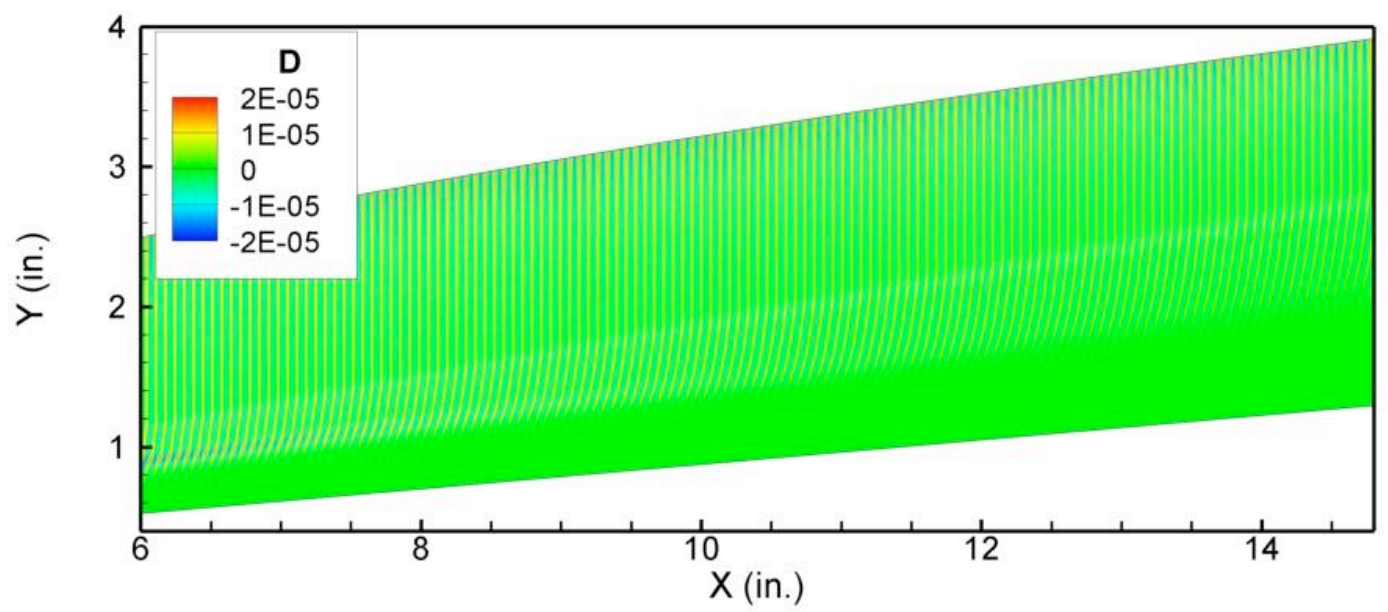

(b) $x=[6.0-15.0]$ in

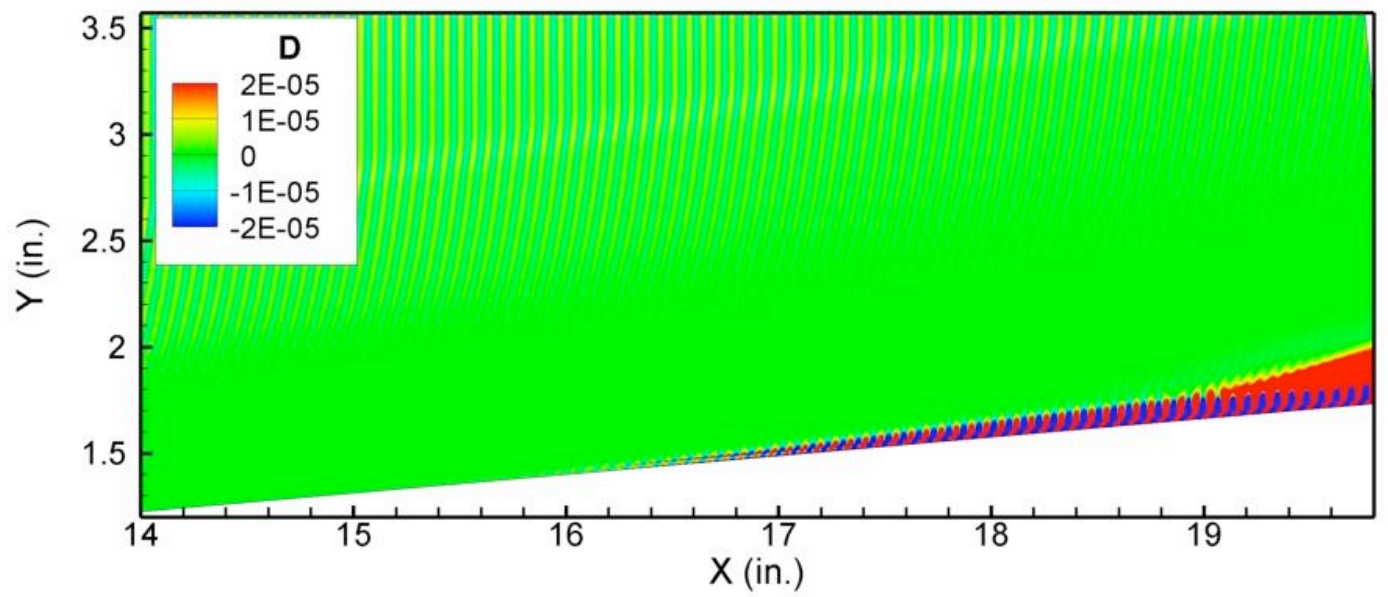

(c) $x=[14.0-19.8]$ in

Figure 20. Contours of unsteady density fluctuations inside the boundary layer along the cooled cone wall $\left(T_{w}=T_{a w}{ }^{\star} 0.20\right),(a) x=[4.0-5.7]$ in, (a) $x=[6.0-15.0]$ in, and (a) $x=[14.0-19.8]$ in. 